\title{
Die Polymorphie des Sacre du printemps in Musik- und Tanzwissenschaft
}

Es ist wohl den Ballets Russes und deren Impresario Sergej Djagilev zu verdanken, dass Igor' Stravinskij 1910 gleichsam über Nacht zu Weltruhm gelangte, und auch sein nachmalig bekanntestes Werk hat seine Entstehung mitunter seiner Begegnung mit Djagilev und der Gattung des Balletts zu verdanken: Le Sacre du printemps. ${ }^{27}$ Letzteres feierte am 29. Mai 1913 in Paris als ein Gemeinschaftswerk von Igor' Stravinskij (Libretto und Musik), Vaclav Nižinskij (Choreografie) und Nikolaj Rerich (Libretto, Dekor und Kostüm) Premiere ${ }^{28}$ und gilt heute als Meilenstein der Musik- und Tanzgeschichte: Stravinskijs Partitur wird aufgrund ihrer rhythmischen und klanglichen Neuerungen als Schlüsselwerk der Sinfonik des 20. Jahrhunderts gesehen. Und Nižinskijs Choreografie gilt spätestens seit dem choreografischen Rekonstruktionsversuch von Millicent Hodson 1987 als eines der wichtigsten Werke der Tanzhistoriografie - „stellt das Stück [Nižinskijs Choreografie] doch in Frage, was bislang Tradition in Tanz und Theater repräsentierte“29, wie die Tanzwissenschaftlerinnen Gabriele Brandstetter und Gabriele Klein im von ihnen herausgegebenen Sammelband Methoden der Tanzwissenschaft formulieren.

Aufgrund der durchschlagenden Wirkung des Stücks entstand dazu in den vergangenen Jahrzehnten eine nahezu unübersichtlich große Zahl an (kunst-) wissenschaftlichen Untersuchungen - überwiegend aus dem Bereich der Musik- und Tanzwissenschaft. An diesen ebenso umfangreichen wie zahlreich erschienenen Publikationen fällt aber auf, dass die Musikwissenschaft die choreografische Arbeit Nižinskijs grosso modo außen vorlässt und die Tanzwissenschaft Stravinskijs Musik gleichsam übergeht. Nur vereinzelt werden in Sacre-Studien sowohl musikalische als auch tänzerische Aspekte berücksichtigt. Für den Bereich der Musikwissenschaft ist hier beispielhaft Charles M.

\footnotetext{
27 Vgl. Richard Taruskin: „How ,The Rite“ Became Possible“, in: Avatar of Modernity, hg. von Danuser und Zimmermann, S. 380-40o, hier S. 380f.

28 Vgl. hierzu den Wortlaut des Uraufführungsprogramms, abgedruckt in: Danuser und Zimmermann, Avatar of Modernity, S. 417, zitiert in Anm. 3.

29 Gabriele Brandstetter und Gabriele KLEIN: „Bewegung in Übertragung. Methodische Überlegungen am Beispiel von ,Le Sacre du printemps'،, in: Methoden der Tanzwissenschaft. Modellanalysen zu Pina Bauschs, Le Sacre du printemps', hg. von DIEs., Bielefeld 2007, S. 9-29, hier S. 19.
} 
Joseph zu nennen, der in seiner 2011 erschienenen Monografie zu Stravinskijs Balletten erstmals Archivmaterial sowohl zum Tanz als auch zur Musik vereint. ${ }^{30}$ Und aus dem Bereich der Tanzwissenschaft stammend spezialisierte sich Stephanie Jordan auf die Entwicklung choreo-musikalischer Analysemethoden und wendete diese unter anderem auch an ausgewählten Balletten Stravinskijs an. ${ }^{31}$ Ein Musik- und Tanzwissenschaft verbindender Ansatz in der Betrachtung des Balletts im Allgemeinen und des Sacre im Besonderen stellt jedoch noch immer ein Forschungsdesiderat dar. ${ }^{32}$ Ein ambivalenter Gebrauch des Ballettbegriffs sowie unterschiedliche methodische Herangehensweisen der beiden Wissenschaftsdisziplinen verschärfen diese Trennung noch: Musikwissenschaftliche Untersuchungen sowohl aus dem englisch- als auch deutschsprachigen Raum beschäftigen sich im Großen und Ganzen mit Stravinskijs Sacre-Komposition und nähern sich dieser größtenteils aus historiografischer und analytischer Perspektive. Die Tanzwissenschaft hingegen konzentriert sich auf Nižinskijs Sacre-Choreografie sowie später entstandene neue choreografische Versionen anderer Choreografen. Deutschsprachige Arbeiten wählen hierbei überwiegend einen kulturwissenschaftlichen Blickwinkel, während historiografische Arbeiten meist aus dem englischsprachigen Raum stammen; nur einige wenige deutschsprachige Tanzwissenschaftler tragen zu diesen Diskussionen bei. ${ }^{33}$ Zwei drängende Fragen sollen deshalb zu Beginn dieser Arbeit

30 Vgl. Charles M. Jose PH: Stravinsky's Ballets, New Haven 2011.

31 Vgl. Stephanie Jordan: Stravinsky Dances. Re-Visions Across a Century, Alton 2007.

32 Dass die Untersuchung der Beziehung von Musik und Choreografie im Ballett im Allgemeinen ein Forschungsdesiderat darstellt, haben Malkiewicz und Rothkamm im Vorwort des von ihnen herausgegebenen Sammelbands 2007 angemerkt. Vgl. Michael MaLkiewicz und Jörg Roth нamm: „Vorwort“, in: Die Beziehung von Musik und Choreographie im Ballett. Bericht vom Internationalen Symposium an der Hochschule für Musik und Theater Leipzig, 23.-25. März 2006, hg. von DIES., Berlin 2007, S. 7-12, hier S. 7. Rothkamm beschäftigte sich während eines Forschungsprojekts zur Ballettmusik im 19. und 20. Jahrhundert unter anderem auch mit dem Sacre. Er veröffentlichte im Rahmen seiner dazu erschienenen Monografie ein Kapitel, in dem er unter anderem die choreografischen Eintragungen eines vierhändigen Sacre-Klavierauszugs untersucht, der sich in der Basler Paul Sacher Stiftung befindet. Vgl. Jörg Rотнкамм: Ballettmusik im 19. und 20. Jahrhundert. Dramaturgie einer Gattung, Mainz u.a. 2011, S. 209-240. Zum Klavierauszug vgl. Anm. 77 .

33 Vgl. hierzu Marion KANT: „Ein Plädoyer für die Tanzgeschichte. Identitätsproblematiken einer Wissenschaftsdisziplin“, in: Tanz. Politik. Identität (Jahrbuch Tanzforschung, Bd. 11), hg. von Sabine KаRоß und Leonore Welzin, Hamburg 2001, S. 81-102. Ein wesentlicher Grund für die kulturwissenschaftliche Orientierung der deutschsprachigen Tanzwissenschaft ist sicherlich in der Entstehungsgeschichte des Faches auszumachen. Die Tanzwissenschaft ist eine sehr junge, aus der Germanistik und Theaterwissenschaft hervorgegangene akademische Disziplin, die sich erst in den 8oer-Jahren des 20. Jahrhunderts in Deutschland zu etablieren begann und stark von den Cultural Studies 
erörtert werden: Was genau ist gemeint, wenn man im wissenschaftlichen Kontext von der Gattung des Balletts oder von Ballett spricht? Und welche Auswirkungen hat dies wiederum auf die wissenschaftliche Betrachtung des Sacre?

Vergleicht man die vielseitige Verwendung des Ballettbegriffs im musik- und tanzwissenschaftlichen Kontext, wird schnell deutlich, dass sowohl sprachliche als auch fachliche Unterschiede eine eindeutige Definition nicht zulassen. Dies konstatierte bereits der österreichische Theaterwissenschaftler und Schriftsteller Joseph Gregor im Vorwort seiner 1944 erschienenen Kulturgeschichte des Balletts:

beeinflusst wurde. Für Beiträge zu einer kulturwissenschaftlich geprägten Tanzwissenschaft vgl. unter anderem Gabriele Brandstette R: „Tanzwissenschaft im Aufwind. Beitrag zu einer zeitgenössischen Kulturwissenschaft", in: Theater der Zeit 12 (2003), S. 4-12; Gabriele Brandstetter: „Tanz als Wissenskultur. Körpergedächtnis und wissenstheoretische Herausforderung", in: Wissen in Bewegung. Perspektiven der künstlerischen und wissenschaftichen Forschung im Tanz, hg. von Sabine Gehm, Pirkko Husemann und Katharina von WiLCKE, Bielefeld 2007, S. 37-48; Gabriele KLEIN: „Tanz in der Wissensgesellschaft", in: Wissen in Bewegung, hg. von Gehm, Husemann und von Wilcke, S. 25-36. Eine der wenigen Ballets-Russes-Historiografen im deutschsprachigen Raum ist Claudia Jeschke. Sie war unter anderem an einer Rekonstruktion der L'aprés-midi d'un Faune-Choreografie von Vaclav Nižinskij beteiligt und ist Mitherausgeberin eines zum 10o. Jubiläum der Ballets Russes erschienenen Ausstellungskatalogs, in dem sie unter anderem lange unveröffentlichtes Material aus russischen Archiven veröffentlichte. Ihre Forschungsarbeit bildet einen Gegensatz zu den kulturwissenschaftlich ausgerichteten Studien um Gabriele Brandstetter. Für Veröffentlichungen Claudia Jeschkes zu den Ballets Russes vgl. unter anderem Claudia JEsch Ke und Nicole Haitzinger (Hg.): Schwäne und Feuervögel. Die Ballets Russes 1909-1929. Russische Bildwelten in Bewegung, Leipzig 2009; Claudia Jeschke, Ursel Berger und Birgit Zeidler (Hg.): Spiegelungen. Die Ballets Russes und die Künste, Berlin 1997; Claudia Jeschke und Ann Hutchinson Guest: Nijinsky's ,Faune' Restored. A Study of Vaclav Nijinsky's 1915 Dance Score, L'après-midi d'un faune' and his Dance Notation System, Philadelphia 1991; Claudia Jeschke (u.a.) unter der Leitung von Jean-Michel Nectoux: Nijinsky. Préludes à ,l'après-midi d'un fauné, Paris 1989. Die Tatsache, dass deutschsprachige Studien im Allgemeinen und kulturwissenschaftliche und historiografische Untersuchungen zum Tanz im Besonderen im englischen Sprachraum nur selten wahrgenommen werden (worauf beispielsweise Marc Franko 2015 im Herausgebervorwort anlässlich der englischen Übersetzung von Gabriele Brandstetters 1995 in Deutschland erschienener Habilitationsschrift Tanz-Lektüren hinwies), verschärft jenes intradisziplinäre Schisma. Vgl. Gabriele BrAndstette R: Poetics of Dance. Body, Image, and Space in the Historical Avant-Gardes, New York 2015. 
Jeder Mensch verbindet eine bestimmte Vorstellung mit dem Klange des Namens Ballett. Ein kurzes, sich anknüpfendes Gespräch belehrt uns bald, daß diese Vorstellungen auch fast bei jedem Menschen andere sind. Der eine versteht darunter eine Gruppe von Tänzern und Tänzerinnen, zusammengehörend und gemeinsam zu ihren Aufgaben geschult. Hier tritt das Ballett ein, heißt es in der Sprache des Theaters. Der zweite, mehr Augenmensch, denkt an Kostümformen, kurze, steif und weiß abstehende Röckchen und an bestimmte Körperhaltungen, vor allem an den Stand und die Fortbewegung auf der Fußspitze. Hier hat sich ein Stil, der einige Generationen vor uns vorherrschend war, zum Begriffe verdichtet, er hat sogar in den berühmten Bildern von Degas seine feststehende Ausprägung, sein malerisches Symbol gefunden, ohne natürlich damit den Begriff anders als einseitig zu erfüllen. Der dritte meint einen Tanz, ein Musikstück, wenn er vom Ballett spricht, und bekräftigt damit als Ohrenmensch die starke Komponente der Musik, die natürlich auch ihrerseits den Begriff lange nicht ausmacht. Man könnte noch lange fortsetzen, man würde vom Ballett $[\mathrm{t}]$ ext und vom Ballettunterricht, von großen Ballerinen, ja sogar von Ballettdekoration hören, aber niemals würde sich, wie es bei Lyrik oder Plastik ganz selbstverständlich ist, ein einheitlicher Begriff zu dem Worte fügen! ${ }^{34}$

Wie heterogen der Begriff Ballett auch im gegenwärtigen Wissenschaftsdiskurs verwendet wird, zeigt schon ein kursorischer Blick in die beiden Referenzlexika der Musikwissenschaft, die auch zentrale tanzwissenschaftliche Beiträge enthalten: Die Musik- und Tanzhistorikerin Sibylle Dahms definiert in der Musik in Geschichte und Gegenwart (MGG) Ballett als „künstlerisch stilisierte Körperbewegung“35 und meint damit ,jenen Bereich des Schautanzes, der sich ab Ende des 16. Jahrhunderts aus dem Kontext musiktheatralischer Formen entwickelte“36. Am Ende des Abschnitts führt sie an, dass der Terminus außerdem oft für „die Komposition von Ballettmusik“37 verwendet wird sowie umgangssprachlich „die Gesamtheit der professionellen Tänzer bzw. die Ballettkompanie“38 bezeichnet. In erster Linie fasst Dahms unter Ballett also den klassischen Bühnentanz und misst demgegenüber der Verwendung des Begriffs zur Bezeichnung der Ballettmusik oder einer Ballettkompanie weniger Bedeutung bei.

Die in der $M G G$ angedeutete Ambiguität findet sich auch in ihrem englischsprachigen Pendant wieder, dem New Grove Dictionary of Music and Musicians. Vergleichbar mit Dahms möchten Rebecca Harris-Warrick, Noel Goodwin und John Percival ballet zuallererst als einen Tanzstil - „a style of theatrical dancing

34 Joseph Gregor: Kulturgeschichte des Balletts. Seine Gestaltung und Wirksamkeit in der Geschichte und unter den Künsten, Wien 1944, S. 9.

35 Dahms, Woitas, Oberzaucher-Schüller, Bröcker u.a., Art. ,Tanz', Sp. 295.

36 Ebd.

37 Ebd.

38 Ebd. 
that developped in France during the 17th Century“39 - begriffen wissen. Im Unterschied zur $M G G$ weisen die Autoren dann allerdings ferner auf das ballet as genre hin und benennen damit einen Begriff, der spätestens seit Ende des 18. Jahrhunderts in Abgrenzung zur stilisierten Körperbewegung ein „spectacle accomponied by purely instrumental music ${ }^{40}$ bezeichnet. Ihrer Ansicht nach ist das ballet neben dem klassischen Bühnentanz vor allem auch als eine theatrale Gattung zu verstehen - eine Gattung, die aus zwei Komponenten besteht: Musik und Tanz. Es ist wohl diese multimediale Bedeutung, die der deutsche Musikwissenschaftler Wolfgang Marx im Sinn hat, wenn er im (englischsprachigen) Artikel "The Ballet as a ,Genre“" folgende ballet-Definition gebraucht: „Ballet in general can be regarded as a combined genre, being shaped as it is by both dance and music."41 Eine Entsprechung für die sowohl im New Grove als auch von Marx verwendete Formulierung ballet as genre existiert in der $M G G$ nicht. Zwar erwähnt Dahms, dass mit dem Ballett „[i]m Sinne der griechischen musiké [...] Dichtung, Musik und Tanz durch die ihnen gemeinsame Dimension des Rhythmus zu einer Einheit zusammengeführt werden [sollten] “42; einen Abschnitt zum Ballett als Gattungsbezeichnung spart sie jedoch aus, obwohl eine diesbezügliche Verwendung des Terminus im deutschsprachigen Raum durchaus gebräuchlich ist. Dass der Begriff Ballett als Gattungsbezeichnung im deutschprachigen Raum zwar existiert, seine Verwendung allerdings erneut einer gewissen Unschärfe unterliegt, soll im Folgenden beispielhaft anhand zweier Texte aufgezeigt werden: Zum einen handelt es sich um das von Jörg Rothkamm und Michael Malkiewicz verfasste Vorwort des 2006 erschienenen Tagungsbands Die Beziehung von Musik und Choreographie im Ballett, zum anderen um Hermann Danusers Textabschnitt zum „Aufstieg des Balletts“ im für die deutschsprachige Musikwissenschaft maßgeblichen Neuen Handbuch für Musikwissenschaft.

Die Musikwissenschaftler Rothkamm und Malkiewicz folgen in ihrer Gattungsdefinition derselben Stoßrichtung wie der New Grove, wenn sie gleich

39 Rebecca Harris-Warrick, Noël Goodwin und John Percival: Art. ,Ballet', in: The New Grove Dictionary of Music and Musicians, 2. Ausg., hg. von Stanley SAdie, 29 Bde., London 2001 (= The New Grove), Bd. 2, S. 565- 596, hier S. 565.

40 Ebd., S. 566. Zum Ballett als Genre vgl. auch das Kapitel „Ballet from Sixteenth-Century France to Nineteenth-Century Russia; Stravinsky“, in: Richard TARUskin (Hg.): The Oxford History of Western Music, 6 Bde., Oxford 2005, Bd. 4: The Early Twentieth Century, Oxford 2005, S. 131-19o. Er beginnt dieses Kapitel mit einem Abschnitt zu „A Missing Genre“, vgl. ebd., S. 131.

41 Wolfgang MARX: „The Ballet as a ,Genrec. Initial Thoughts on the Generic Identity of a Multimedia Art Form“, in: Die Beziehung von Musik und Choreographie im Ballett, hg. von ders. und Rothkamm, S. 13-26, hier S. 18.

42 Dahms, Woitas, Oberzaucher-Schüller, Bröcker u.a., Art. ,Tanz', Sp. 296. 
zu Anfang anmerken, dass „für die Gattung Ballett von ihren Anfängen bis zur Gegenwart [...] zwei Komponenten essentiell ${ }^{43}$ sind: Musik und Choreografie. Der hier gewählte Begriff Ballett gründet also in einem Verständnis des Balletts als einer theatralen Gattung, die mehrere Kunstformen in sich vereint. Ein solches multimediales Gattungsverständnis ließe sich auch bei Dahms herauslesen, wenn sie beschreibt, dass das Ballett aus den Bestandteilen „Dichtung, Musik und Tanz" entstanden sei - eine Sichtweise, die so auch in tanzwissenschaftlichen Lexika zu finden ist. ${ }^{44}$ Danuser hingegen weicht von diesem theatralen oder multimedialen Gattungsverständnis deutlich ab, wenn er vermerkt, dass das „Ballett [...] um 1910 [...] zu einer charakteristischen Gattung der Neuen Musik“ ${ }^{45}$ wurde. Auch wenn er in der Folge mehrmals zwischen ballett- und musikgeschichtlichen Bestrebungen unterscheidet und damit deutlich macht, dass für ihn eine Ballettgeschichte als eine Geschichte des (klassischen Bühnen-)Tanzes klar von einer (Ballett-)Musikgeschichte abzugrenzen ist, fällt auf, dass er an einigen zentralen Stellen mit Ballett nicht etwa eine theatrale, sondern eine musikalische Gattung benennt. ${ }^{46}$ Ein Standardwerk der (deutschsprachigen) Musikwissenschaft verwendet die Gattungsbezeichnung Ballett also unter anderem synonym mit Ballettmusik. Und so ist festzuhalten, dass der Ballettbegriff - zumindest im deutschsprachigen Kontext - nicht nur als Bezeichnung der Ballettmusik verwendet wird, sondern auch als Bezeichnung des Balletts im Sinne einer sowohl theatralen als auch musikalischen Gattung. ${ }^{47}$

43 Malkiewicz und Rothkamm, „Vorwort“, S. 7.

44 Vgl. hierzu Debra Craine und Judith Mackrell: Art. ,Ballet', in: The Oxford Dictionary of Dance, 2. Aufl., hg. von DiEs., Oxford 2000, S. 4O-41, hier S. 40: „[Ballet is] [a] form of Western academic theatrical dance based on the technique known as danse d'école (the classical school), usually presented with elements of music and design to dramatic or lyric effect."

45 Hermann Danuser (Hg.): Die Musik des 20.Jahrhunderts (Neues Handbuch der Musikwissenschaft, hg. von Carl DAHLHAUs, fortgeführt von Hermann DANUSER, 13 Bde., Wiesbaden und Laaber 1980-1992), Bd. 7, Laaber 1984, S. 65.

46 Vgl. unter anderem Danuser, Die Musik des 20. Jahrhunderts, S. 72: „Die Nobilitierung des Balletts als Kompositionsgattung zeitigte ferner für die Geschichte der musikalischen Form Konsequenzen, die denen im Bereich des Stils nicht nachstanden." Oder ebd., S. 77 : „[...] so zeigt nichts deutlicher die tiefgreifende Wandlung des Balletts: Einst als funktionale Gattung für die Kompositionsgeschichte irrelevant, ist es in der Neuen Musik des 2o. Jahrhunderts zu einem Problembereich autonomen Komponierens geworden."

47 Sibylle Dahms' in der $M G G$ dargelegter Feststellung, dass der Ballettbegriff auch zur Bezeichnung der Ballettmusik verwendet wird, muss daher hinzugefügt werden, dass er ebenso als Gattungsbegriff gebräuchlich ist - und zwar sowohl für die Bezeichnung der theatralen bzw. multimedialen Gattung Ballett als auch für die musikalische 
Dies alles wirkt sich freilich auch auf die wissenschaftliche Betrachtung des Gegenstands aus: Eine Ballettforschung als eine Wissenschaft, die alle dem Ballettbegriff inhärenten Ausprägungen gleichberechtigt berücksichtigt, existiert bis dato nicht. Unterschiedliche Fachdisziplinen - allen voran Musikund Tanzwissenschaft - haben in den vergangenen Jahrzehnten verschiedene Methoden entwickelt, um sich dem Ballett anzunähern. Der Untersuchungsgegenstand selbst aber ist divergent, was auch auf die terminologische Unschärfe zurückzuführen ist: Im musikwissenschaftlichen Kontext wird Ballett zur Bezeichnung der musikalischen Gattung Ballett und ebenso für die der (Ballett-)Musik verwendet, die sich im Laufe des 2o. Jahrhunderts von einer zunächst funktionalen Gattung der Tanzmusik zu einer autonomen Gattung emanzipierte und daraufhin vermehrt zum Gegenstand musikwissenschaftlicher Betrachtung wurde. ${ }^{48}$ In tanzwissenschaftlichen Untersuchungen bezeichnet Ballett hingegen hauptsächlich den in Ballettdefinitionen meist zuerst genannten (künstlerischen) Bühnentanz bzw. die für das Ballett entworfene Choreografie. Ballett als theatrale Gattung wird noch immer zu selten Gegenstand musik- oder tanzwissenschaftlicher Betrachtung. ${ }^{49}$ Im Folgenden

Gattung Ballett, um ebendiese (Ballett-)Musik innerhalb des Gefüges der musikalischen Gattungen zu kategorisieren.

48 Die Idee der Selbstzweckhaftigkeit von Musik, die sich hinter den Begriffen autonome bzw. absolute Musik verbirgt, wurde erstmals um 1800 formuliert und meint damit spätestens ab der Mitte des 19. Jahrhunderts jene Musik, die von jeglichen außermusikalischen Faktoren unabhängig ist und sich daher allein durch sich selbst begründet. Diese Idee prägte bis in die Mitte des 20. Jahrhunderts die Vorstellung dessen, was Musik ist. Vgl. Wilhelm SEIDE L: Art. ,Absolute Musik', in: $M G G$ 2, Sachteil: Bd. 1, Kassel u.a. 2001, Sp. 15-24, hier Sp. 15f.; vgl. hierzu auch Jin-Ah KIM: „Mimesis und Autonomie. Zur Genese der Idee der ,autonomen Musik'“, in: Die Musikforschung 64/1 (2011), S. 24-45. Die Musikwissenschaft bzw. die Musikgeschichtsschreibung ist in der Tradition der Autonomieästhetik zu sehen. Vgl. hierzu den Aufsatz von Tobias JANZ: „Musikwissenschaft als Kunstwissenschaft?“, in: Historische Musikwissenschaft. Grundlagen und Perspektiven, hg. von Michele Calella und Nikolaus Urbane K, Stuttgart und Weimar 2013, S. 56-81, hier S. 72-76. Carl Dahlhaus widmete beispielsweise Entstehung und Geschichte der Idee der absoluten Musik eine eigenständige Studie. Vgl. Carl Dahlhaus: Die Idee der absoluten Musik, Kassel 1978. Zur Autonomieästhetik in der Kunst allgemein vgl. unter anderem Michael Müller u.a.: Autonomie der Kunst. Zur Genese und Kritik einer bürgerlichen Kategorie, Frankfurt a.M. 1972. Als funktionale Musik (oder auch Gebrauchsmusik) gelten und galten hingegen jene „Arten [...] [von] Musik, [...] die nicht sub specie des Begriffs des Artificiums und Kunstwerks, des musikalisch Artifiziellen und Autonomen sich begreifen und zu begreifen sind“. Vgl. Hans Heinrich EgGebrecht: „Funktionale Musik“, in: Archiv für Musikwissenschaft 30/1 (1973), S. 1-25, hier S. 1.

49 Beispielhaft für wissenschaftliche Arbeiten über das Ballett, in denen sowohl Tanz als auch Musik untersucht werden, sind folgende Arbeiten: Roland John WiLEY: Tchaikovsky's Balletts. ,Swan Lake؛. ,Sleeping Beauty؛ ,Nutcrucker', Oxford 1985; Charles M. JosePH: 
soll das Ballett im Allgemeinen und dasjenige Stravinskijs im Besonderen allerdings in genau diesem theatralen bzw. multimedialen Verständnis betrachtet werden: als ein gleichberechtigtes Zusammenspiel von Tanz und Musik (und gegebenenfalls Bühnenbild).

1.2 Igor' Stravinskijs Ballett(musik) in Musik- und Tanzwissenschaft Nicht allein der Begriff Ballett ist unscharf definiert, auch inhaltlich wird die Gattung Ballett stiefmütterlich behandelt. So spielte Ballettmusik für die Musikwissenschaft bisher eher eine untergeordnete Rolle; wegen ihrer Funktionsgebundenheit wurde ihr im Laufe des 19. Jahrhunderts oftmals der künstlerische Wert abgesprochen. So werden beispielsweise auch heute noch die Ballettkomponisten Léon Minkus, Cesare Pugni oder Riccardo Drigo als Vertreter eines Komponistentypus beschrieben, dessen Musik, wie die Theater- und Tanzwissenschaftlerin Gunhild Oberzaucher-Schüller in der $M G G$ resümiert, „allein als rhythmisches Raster für den Tanz konzipiert“50 wurde - eine Eigenschaft, die jene Künstler für die musikwissenschaftliche Forschung bis heute als uninteressant kategorisiert. Die Ballettmusik Adolph Adams, Léo Délibes' oder Pëtr Čajkovskijs kann allerdings keinesfalls als ein solches bloßes „Raster für den Tanz" abgetan werden. Immerhin entwickeln sich hier "[m] usikalische Motive [...] in Anlehnung an sinfonische und musikdramatische Kompositionstechniken aus den verwendeten Themen selbst" ${ }^{41}$. Dennoch ereilte sie, was die musikwissenschaftliche Nichtbeachtung angeht, ein ähnliches Schicksal. Als beispielhaft für jene lang anhaltende Geringschätzung der Ballettmusik mag die Tatsache angeführt sein, dass im 1988 begründeten Handbuch der musikalischen Gattungen erst 2009 ein Band zum Thema Gesellschaftsmusik, Bläsermusik, Bewegungsmusik erschien, der lediglich unter dem Teilkapitel Bewegungsmusik einige Aufsätze zum Ballett

Stravinsky and Balanchine. A Journey of Invention, New Haven 2002; Joseph, Stravinsky's Ballets; Jordan, Stravinsky Dances; Simon Morrison: „The Origins of ,Daphnis and Chloé (1912)“, in: 19th Century Music 28/1 (2004), S. 50-76; Tamara Levitz: „Syvilla Fort's Africanist Modernism and John Cage's Gestic Music. The Story of ,Bacchanale'،, in: South Atlantic Quaterly 104/1 (2005), S. 123-149; Deborah MAWER: The Ballets of Maurice Ravel. Creation and Interpretation, Aldershot 2006; Davinia CADDY: The Ballets Russes and Beyond. Music and Dance in Belle-Epoque Paris, Cambridge 2012; Wayne Heisler Jr.: The Ballet Collaborations of Richard Strauss, Rochester 20o9; Tamara Levitz: Modernist Mysteries. Perséphone, New York 2012. Immerhin ist mit Davinia Caddy festzustellen, dass Tanz innerhalb der Musikwissenschaft zum Gegenstand einer wachsenden Anzahl von Artikeln, Tagungsbeiträgen und Sonderausgaben wird. Vgl. Caddy, The Ballets Russes and Beyond, S. 20.

$5^{\circ}$ Dahms, Woitas, Oberzaucher-Schüller, Bröcker u.a., Art. ,Tanz', Sp. 327.

$5^{1} \quad$ Vgl. ebd., Sp. 328. 
enthält.52 In seinem dort publizierten Aufsatz „Zur Kompositionsstruktur von Ballettmusik" spricht der Musikwissenschaftler Thomas Steiert das verhaltene Ballettinteresse seiner Fachkollegen auch explizit an. ${ }^{53}$

Bis heute fehlt es folglich an genuinem (musik-)wissenschaftlichem Interesse am Ballett als musikalischem Phänomen. ${ }^{54}$ Eine Ausnahme bilden Ballettwerke, die neben ihrer tanzgebundenen Form von Anfang an auch autonom, das heißt konzertant und somit frei vom tänzerischen Geschehen, aufgeführt wurden. So begreift die Musikwissenschaft die Ballette Stravinskijs - insbesondere seine drei frühen russischen: L'Oiseau de feu, Pétrouchka und Le Sacre du printemps - gemeinhin als Schlüsselwerke, denn neben der Bühnenform wurden sie kurz nach ihrer theatralen Uraufführung immer auch konzertant aufgeführt, ohne dass „das Wegfallen der Choreographie als Manko empfunden “55 worden sei, wie der russisch-schweizerische Musikwissenschaftler und Stravinskij-Zeitgenosse Jacques Handschin 1933 in seinem Versuch einer Einführung über den Komponisten vermerkte. Die Tatsache, dass Ballettmusik mit Beginn des 20. Jahrhunderts zunehmend autonom aufgeführt wurde, schaffte erst die Voraussetzung dafür, dass die Musikwissenschaft sich mit dieser Gattung auseinanderzusetzen begann. Diese neue Gewichtung äußert sich zum Beispiel im 1979 begründeten Neuen Handbuch der Musikwissenschaft. Während die Ballettmusik im sechsten Band der Reihe (Die Musik des 19. Jahrhunderts) gar keine Erwähnung findet - und dass, obwohl das klassische Ballett genau in jenem Jahrhundert seinen Höhepunkt fand ${ }^{56}{ }_{-}$,

52 Vgl. Siegfried MaUser und Elisabeth Schmierer (Hg.): Gesellschaftsmusik, Bläsermusik, Bewegungsmusik (Handbuch der Musikalischen Gattungen, 17 Teile in 24 Bde., hg. von Siegfried MAUSER, Laaber 1993-2010, Bd. 17.1), Laaber 2009.

53 Vgl. Thomas Steiert: „Zur Kompositionsstruktur von Ballettmusik“, in: Gesellschaftsmusik, Bläsermusik, Bewegungsmusik, hg. von Mauser und Schmierer, S. 225-231, hier S. 225 .

54 Für deutschsprachige musikwissenschaftliche Beiträge zur Ballettforschung vgl. unter anderem Malkievicz und Rothkamm, Die Beziehung von Musik und Choreographie im Ballett; Konrad LANDRE H: Manuel de Fallas Ballettmusik, Laaber 2004; Rothkamm, Ballettmusik im 19. und 20. Jahrhundert; Steffen Sс нміDт: Musik der Schwerkraft. Die Beziehung von Musik und Ballett in Deutschland nach 1945. Dargestellt am Werk Bernd Alois Zimmermanns, Berlin 2012; Monika Wortas: Geschichte der Ballettmusik. Eine Einführung, Laaber 2018.

55 Jacques Handschin: Igor Strawinsky. Versuch einer Einführung (121. Neujahrsblatt der allgemeinen Musikgesellschaft in Zürich), Zürich und Leipzig 1933, 38 S., hier S. 15f., abgedruckt in: Strawinsky. Sein Nachlass. Sein Bild, hg. vom KunStMUSEUM BASEL und der Paul Sacher Stiftung Basel, Basel 1984, S. 187-225.

$5^{6}$ Zur Bedeutung der Čajkovskij-Ballette für das klassische Ballett und zur Rolle des Choreografen Marius Petipa sowie zur Sonderrolle Russlands für das Ballett im späten 19. Jahrhundert vgl. Dahms, Woitas, Oberzaucher-Schüller, Bröcker u.a., Art. ,Tanz', Sp. 326; 
wird ihr im siebten Band (Die Musik des 20. Jahrhunderts) sogar eine zentrale Rolle für die Musikgeschichtsschreibung zugesprochen. ${ }^{57}$ Dem Aspekt des Tanzes wird allerdings wenig bis gar keine Aufmerksamkeit zuteil.

Möglicherweise war es diese stiefmütterliche Behandlung der Ballettmusikgeschichte aufseiten der Musikwissenschaft, die dazu geführt hat, dass die Geschichte des Balletts heutzutage größtenteils durch Tanzwissenschaftler erzählt wird: als eine Geschichte des Tanzes, in der die zugehörige Musik so gut wie immer übergangen wird. ${ }^{5}$

Da sowohl in der Musik- als auch der Tanzwissenschaft Ballettwerke des 2o. Jahrhunderts im Allgemeinen, diejenigen Stravinskijs im Besonderen, nur sporadisch als ein gemeinsames Ganzes von Musik, Tanz und Bühnenbild begriffen werden, herrscht in der wissenschaftlichen Betrachtung dieser multimedialen Kunstform folglich überwiegend eine systematische Trennung von musikalisch Notiertem, Erklingendem und tänzerisch bzw. szenisch Visualisiertem, Performativem vor. Dieses Schisma wird noch dadurch forciert, dass innerdisziplinäre Diskurse sich oft grundlegend von denjenigen der jeweils anderen Disziplin unterscheiden.

Es ist nur verständlich, dass diese forschungsgeschichtlichen Hintergründe tief greifende Auswirkungen auf die Betrachtung von Stravinskijs Sacre hatten. Und so drängt sich die Frage auf, inwiefern die Erkenntnisse der einzelnen Wissenschaftsdisziplinen sinnvoll miteinander in Beziehung gesetzt werden können.

Taruskin, The Oxford History of Western Music, Bd. 4, S. 138-146. Eine umfangreiche Untersuchung von Čajkovskijs Balletten bietet Wiley, Tchaikovsky's Ballets. Möglicherweise aufgrund der Sonderrolle des 19. Jahrhunderts für das Ballett legen jüngst erschienene Ballettstudien eine vergleichsweise größere Aufmerksamkeit auf jenes Jahrhundert. Vgl. unter anderem Marian Sмітн: Ballet and Opera at the Age of ,Giselle; Princeton und Oxford 200o; Wiley, Tchaikovsky's Ballets; Knut Arne Jürgensen: The Verdi Ballets, Parma 1995; Rothkamm, Ballettmusik des 19. und 20. Jahrhunderts; Malkievicz und Rothkamm, Die Beziehung von Musik und Choreographie im Ballett; Marion Kant (Hg.): The Cambridge Companion to Ballet, Cambridge u.a. 2007.

57 Vgl. hierzu Danuser, Die Musik des 20. Jahrhunderts, S. 62-77.

$5^{8}$ Viel zitierte historische Referenzbücher zum Ballett konzentrieren sich fast ausschließlich auf eine reine Beschreibung des tänzerischen Aspekts der Werke. So auch folgende Übersichtswerke: Sibylle DAhms in Zusammenarbeit mit Claudia Jesch KE und Monika Woitas (Hg.): Tanz, Kassel 20o1; Garafola, Diaghilev's Ballets Russes; Hartmut Regitz, Otto Friedrich Regner und Heinz-Ludwig Schneiders: Reclams Ballettführer, 11. Aufl., Stuttgart 1992; Jochen ScнміDт: Tanzgeschichte des 20.Jahrhunderts in einem Band, Berlin 2002; Kant, The Cambridge Companion to Ballet; Jennifer Homans: Apollo's Angels. A History of Ballet, New York 2010. Theoretische Werke zum Tanz im Allgemeinen betrachten das klassische Ballett nur am Rande als eine von vielen Ausformungen des künstlerischen Bühnentanzes. 


\section{Der Sacre in Musik- und Tanzwissenschaft}

2.1 Innerdisziplinäre Sacre-Diskurse der Musikwissenschaft

Wenn der amerikanische Literaturwissenschaftler Daniel Albright in seinen Bemerkungen $\mathrm{zu}$ einem musikalischen Primitivismus ${ }^{59}$ in der Uraufführung des Sacre am 29. Mai 1913 "the defining Moment of Modernism [...] in music" 60 sieht und das Werk im selben Abschnitt als "the most famous of all Primitive experiments "61 beschreibt, stellt er damit zwei Konstanten heraus, die sich in ihrem Dualismus wie ein Grundmotiv durch die musikwissenschaftliche Sacre-Rezeption ziehen: Zum einen verweist er auf die radikale Modernität der Musik des Balletts, zum anderen auf die ihr entgegenstehende, hervorstechende ,Primitivität' des Sujets. Diese Dualität hatte der russische Musikwissenschaftler Michail Druskin bereits 1976 mit den „beiden Intonationspole[n] [...] Frühgeschichtliches, Altertümliches, Archaisches und Zeitgenössisches, Alltägliches" ${ }^{42}$ beschrieben. Beim Schweizer Musikwissenschaftler Theo Hirsbrunner findet sich 1982 ebenfalls ein Hinweis auf die Janusköpfigkeit des Sacre zwischen Primitivität und Moderne, wenn er konstatiert, dass „[d]ie Reduktion der Melodien auf einige tatsächlich sehr primitiv wirkende Formeln [...] längst wettgemacht [wird] durch die äußerst kunstvolle rhythmische Struktur und das Raffinement der Orchestration, das weit über das von Rimsky und Debussy Gewohnte hinausgeht"63. Wolfgang Dömling schließt sich all dem an, wenn er im selben Jahr beschreibt, wie im Sacre das „älteste Sujet"64 - nämlich „die barbarische Welt des heidnischen Russland [...] - die neueste Musik“65 erzeugen würde.

Im Kontext dieses Spannungsfelds zwischen Primitivismus und Moderne werden häufig auch die Ausführungen Richard Taruskins gelesen. ${ }^{66}$ Für den

59 Zum Primitivismusbegriff im Allgemeinen und zu seiner Verwendung in Musik- und Tanzwissenschaft im Besonderen vgl. Kapitel I.2.3.

6o Daniel Albrig ht: Modernism and Music. An Anthology of Sources, Chicago und London 2004, S. 236 .

61 Ebd.

62 Michail Druskin: Igor Strawinsky. Persönlichkeit. Schaffen. Aspekte, hg. und übertr. aus dem Russischen von Christof RÜGER, Leipzig 1976, S. 63.

63 Theo Hirsb runne r: Igor Strawinsky in Paris, Laaber 1982, S. 48.

64 Wolfgang DömLING: Igor Strawinsky. In Selbstzeugnissen und Bilddokumenten, Hamburg 1982, S. 29 .

65 Ebd.

66 Taruskin selbst verwendet den Primitivismusbegriff in seinen maßgeblichen Texten zum Sacre allerdings nur äußerst selten. Vgl. hierzu zum Beispiel Taruskin, Stravinsky and the Russian Traditions, Bd. 1, S. 85of.; Richard TARUSkin: „Stravinsky and the Subhuman. A Myth of the Twentieth Century. The Rite of Spring, the Tradition of the New, and the 
amerikanischen Musikwissenschaftler und Stravinskij-Experten besteht kein Zweifel: Ohne ein Zusammentreffen mit Djagilev und dessen Umfeld um 1909 hätte Stravinskij den Sacre nie geschrieben. ${ }^{67}$ In seiner 1996 erschienenen Monografie zu den russischen Werken des Komponisten (Stravinsky and the Russian Traditions) zeichnet Taruskin ausführlich den Einfluss der russischen (Volks- und Kunstmusiks-)Tradition und Kunst auf Stravinskijs musikalisches Schaffen nach und vertritt die These, dass die Ursprünge der im Sacre sich manifestierenden radikalen Modernität auch ebendort zu lokalisieren seien $^{68}$ - ein Einfluss, den der Künstler selbst zeitlebens mehr oder weniger bewusst verschleiert und den die Forschung bis Erscheinen jener monumentalen Studie nicht in dieser Konsequenz wahrgenommen hat. ${ }^{69}$ Taruskin beschreibt en détail, wie Stravinskij durch Djagilevs Auftragskompositionen für die Ballets Russes 1909/10 mit ästhetischen Perspektiven in Kontakt kam, zu denen er in seinem bisherigen Sankt Petersburger Umfeld keinen Zugang hatte. ${ }^{70}$ Er zeigt auf, wie sich die Begegnung Stravinskijs mit den Ballets Russes und seine intellektuelle Beschäftigung mit deren Ideengut unmittelbar auf sein kompositorisches Schaffen auswirkten. Durch die detailreiche Aufarbeitung russischsprachiger Quellen, die der westlichen Forschung bis dahin größtenteils unbekannt waren, kam Taruskin bezüglich der Entstehungsumstände des Sacre zu Erkenntnissen, die den musikhistoriografischen Diskurs seitdem entscheidend prägen: ${ }^{71}$ So konnte er zum Beispiel nachweisen, dass sich Stravinskij für seine Sacre-Komposition mit archaisch

Music itself“', in: Defining Russia Musically. Historical and Hermeneutical Essays, hg. von DERS., Princeton und Oxford 1997, S. 36o-388, hier S. 378-38o.

67 Vgl. Taruskin, „How ,The Rite` Became Possible“, S. 385.

68 Vgl. Taruskin, Stravinsky and the Russian Traditions. Inwiefern Stravinskij in seinen Kompositionen der russischen Tradition verpflichtet war und inwieweit diese sein künstlerisches Schaffen prägte, zeigt neben Taruskins umfangreicher Werkmonografie auch Svetlana Savenkos ausführliche Skizzenstudie. Vgl. Svetlana SAvEn Ko: Mir Stravinskogo, Moskau 2001. Zu den von Taruskin dargelegten Einflussfaktoren auf Stravinskijs musikalisches Schaffen vgl. Kapitel II.

69 Stravinskij verleugnete beispielsweise zeitlebens den Rückgriff auf Volksliedmaterial im Sacre, obgleich er nachweislich solches verwendet hatte. Vgl. Taruskin, Stravinsky and the Russian Traditions, Bd. 2, S. 891; zum Stravinskij'schen ,Amnesie'-Phänomen allgemein vgl. unter anderem ders., „Stravinsky and the Subhuman“, S. 364f.; DERS.: „Stravinsky and the Traditions. Why the Memory Hole?", in: Opus 3/4 (1987), S. 10-17.

$70 \quad$ Vgl. Taruskin, Stravinsky and the Russian Traditions, Bd. 1, S. 423-553.

71 Vgl. ebd., Bd. 1 und 2, S. 849-1033. Einige Texte der bis dato entstandenen musikwissenschaftlichen Sacre-Forschungsliteratur befinden sich unter anderem bei ders., „Resisting ,The Rite“', S. 417 f. oder bei Jonathan BERnARD: „Le Sacre` Analysed“, in: Avatar of Modernity, hg. von Danuser und Zimmermann, S. 284-305. 
gedachten Riten ${ }^{72}$ und den damit in Zusammenhang stehenden (russischen) Volksliedern beschäftigte und ebendiese auch in seiner Komposition verarbeitete. ${ }^{73}$ Ferner unterstreicht Taruskin die Wichtigkeit der tänzerischen Aktion auf der Bühne für die Entstehung des Werkes, indem er deutlich macht, dass Stravinskij die tänzerische Aktion bereits in der Ausarbeitung des Librettos mitdachte. ${ }^{74}$

Taruskins Erkenntnisse gelten vielen Stravinskij-Forschern als kanonisch. Und so plädiert beispielsweise auch der amerikanische Musikwissenschaftler Charles Joseph dafür, dass der Sacre in der ursprünglichen Form als Ballett betrachtet werden muss. Er geht dabei sogar einen Schritt weiter als Taruskin und berücksichtigt für seine Studie neben musikalischen auch tänzerische Quellen. Joseph belässt es zwar bei einem rein historiografischen Beschreiben der Entstehungsumstände, verdeutlicht aber auch, welche zentrale Rolle der Tanz in der Entstehung des Balletts hatte. ${ }^{75} \mathrm{Jörg}$ Rothkamm stellt in seiner Monografie Ballettmusik im 19. und 20. Jahrhundert die Frage, ob Stravinskij den Sacre „von vornherein choreographisch-musikalisch geplant [hatte] und - falls ja - inwieweit Nižinskij dies umsetzte" ${ }^{\text {"76 }}$, und zieht vor allem die choreografischen Anmerkungen zurate, die sich in Stravinskijs Handexemplar des vierhändigen Sacre-Klavierauszugs befinden. ${ }^{77}$ Er kommt aber zu dem Schluss, dass über

72 Im 19. Jahrhundert wurden in Russland zahlreiche Sammlungen zu archaischen Riten, Bräuchen und Volksgesängen veröffentlicht. Damals war es gängige Praxis, von bäuerlicher Folklore auf heidnische Riten zu schließen. Um 1900 galten diese Sammlungen als Quelle urzeitlicher Riten und Bräuche und waren bei vielen Künstlern sehr begehrt - so auch bei den Mitgliedern der Mir Iskusstva bzw. der späteren Ballets Russes. Sie werden im Folgenden als ,archaisch gedachte 'Riten, Bräuche oder Volksgesänge bezeichnet.

73 Zuallererst machte Lawrence Morton auf die Verwendung von Volksliedmaterial im Sacre aufmerksam. Vgl. Lawrence Morton: „Footnotes to Stravinsky Studies. ,Le Sacre du printemps'،, in: Tempo 128 (1979), S. 9-16. Taruskins als kanonisch geltender Artikel hierzu erschien 1980. Vgl. Richard TARuskin: „Russian Folk Melodies in ,The Rite of Spring““, in: Journal of the American Musicological Society 33/3 (1980), S. 501-543. Dem Artikel folgte die umfangreiche Darstellung in ders., Stravinsky and the Russian Traditions, Bd. 1, S. 849-966.

74 Vgl. unter anderem ebd., S. 865.

75 Vgl. Joseph, Stravinsky's Ballets, S. 89. Auch Tamara Levitz' Arbeit ist von Taruskin beeinflusst; sie kritisiert ihn allerdings dafür, zu theoretisch zu sein. Vgl. Tamara Levitz: „The Chosen One's Choice“, in: Beyond Structural Listening? Postmodern Modes of Hearing, hg. von Andrew Dell'Antonio, Berkeley 2004, S. 70-108.

76 Rothkamm, Ballettmusik im 19. und 20. Jahrhundert, S. 212.

77 Der von Rothkamm befragte Klavierauszug befindet sich in der Sammlung Igor Strawinsky der Paul Sacher Stiftung in Basel. Vgl. Igor STRAVinsky: ,Le Sacre du printemps: Tableaux de la russie païenne en deux parties. Réduction pour piano à quatre mains par l'auteur, Berlin, Moskau und Sankt Petersburg 1913. Diese Quelle stand der Choreografin Millicent Hodson bei ihrem Rekonstruktionsversuch der Nižinskij-Choreografie noch nicht 
die Genauigkeit der Zusammenarbeit anhand der zur Verfügung stehenden Quellen nur spekuliert werden kann. ${ }^{78}$

Für andere Stravinskij-Forscher sorgt Taruskins Standpunkt hingegen bis heute für Diskussionen. ${ }^{79}$ Der amerikanische Musiktheoretiker Pieter van den Toorn etwa sieht im Sacre keinesfalls ein Bühnenwerk. Da er bereits kurz nach seiner Uraufführung (ab 1914) äußerst erfolgreich im Konzertsaal aufgeführt wurde und seitdem eine durchschlagende konzertante Rezeptionsgeschichte vorzuweisen hat, hält van den Toorn die von Taruskin herausgestellten russischen Wurzeln des Werkes und seine damit verbundene Verankerung im Ballett für vernachlässigbar. ${ }^{80}$ Jonathan Bernard kommentierte die mittlerweile seit 25 Jahren öffentlich ausgetragene Kontroverse von Taruskin und van den Toorn jüngst wie folgt: „Unless one believes in parallel universes, ,The Rite as Ballet First and Foremost' would seem an unprofitable thesis to pursue. ${ }^{\text {“81 }}$

zur Verfügung. Rothkamm zielt mit seiner musikalisch-choreografischen Analyse deshalb unter anderem auch darauf, die choreografische Realisierung Hodsons zu hinterfragen. Vgl. Rothkamm, Ballettmusik im 19. und 20. Jahrhundert, S. 212-23o. Zum Rekonstruktionsversuch und zu dessen wissenschaftlicher Rezeption vgl. Kapitel I.2.2. sowie Anm. 94.

78 Vgl. Rothkamm, Ballettmusik im 19. und 20. Jahrhundert, S. 233. Rothkamm ist hierin vor allem deshalb zuzustimmen, da die Autorschaft der choreografischen Eintragungen im Basler Klavierauszug bislang nicht geklärt werden konnte. Stravinskij behauptete, dass alle Eintragungen von ihm selbst stammten. Vgl. Igor Stravinsky: „Appendix III: The Stravinsky-Nijinsky Choreography“, in: The Rite of Spring. Sketches 1911-1913. Facsimile Reproductions from the Autograph, hg. von DERS., London u.a. 1969, S. 35-43, hier S. 35. Es befinden sich aber mindestens drei verschiedene Handschriften sowie zusätzliche Eintragungen von Robert Craft im Klavierauszug. Vgl. Rothkamm, Ballettmusik im 19. und 20. Jahrhundert, S. 231. Ulrich Mosch, der bis 2014 die Sammlung Igor Strawinsky der Paul Sacher Stiftung betreute, wies mich dankenswerterweise darauf hin, dass eine genaue zeitliche Zuordnung der Eintragungen bislang noch nicht möglich war.

79 Vgl. hierzu Bernard, „Le Sacre‘ Analysed“, S. 284-305, insbesondere S. 292-295 und 304f.

8o Vgl. Pieter C. VAN DEN TOORN: Stravinsky and, The Rite of Spring: The Beginnings of a Musical Language, Berkeley und Los Angeles 1987, S. 1-22. Viel zitierte und rein analytische Betrachtungen des Sacre finden sich unter anderem auch bei Allen ForTe: The Harmonic Organization of ,The Rite of Spring', New Haven und London 1978; Pierre Boulez: „Stravinsky demeure“, in: Relevés d'apprenti, hg. von Paule Thévenin, Paris 1966, S. 75-145; Olivier Messiaen: Traité de rhythme, de couleur, et d'ornitologie, 7 Bde., Paris 1994-2002, Bd. 2, Paris 1995, S. 91-147; für weitere Beispiele vgl. Arnold Whittall: „Music Analysis as Human Science? ,Le Sacre du printemps' in Theory and Practice“, in: Music Analysis 1/1 (1982), S. 33-53; Arnold WhitTALL: „Defusing Dionysus? New Perspectives on ,The Rite of Spring'“, in: Music Analysis 21/1 (2002), S. 87-104 sowie den 2013 erschienenen Beitrag von Bernard, „Le Sacre' Analysed“, S. 284-305.

81 Ebd., S. 305. Zu Bernards Darstellung der schriftlich ausgetragenen Debatte vgl. ebd., S. 294f. und inbesondere seine Anm. 21. 
Wie in Kapitel I.1.2. dargelegt, gibt es mittlerweile Musikwissenschaftler, die sich der sogenannten Gebrauchsmusik ${ }^{82}$ im Allgemeinen und Ballettmusik im Besonderen zuwenden und dabei ganz selbstverständlich tänzerische Quellen bzw. (soweit verfügbar) im Zusammenhang mit der Choreografie stehende historiografische Fakten mitberücksichtigen. Dennoch bleibt Stravinskijs Sacre als Gegenstand musikwissenschaftlicher Betrachtung sowohl autonomes musikalisches Werk als auch Ballett. Eine Ursache der innerfachlichen Debatte darüber, ob gerade dieses für die Musikgeschichte des 20. Jahrhunderts so zentrale Opus eben nicht als Ballett, sondern als autonomes Werk betrachtet werden sollte, ist sicherlich darin auszumachen, dass Stravinskijs frühe russische Ballette im Allgemeinen und sein Sacre im Besonderen als Prototypen einer neuen Ballettmusik im 20. Jahrhundert begriffen wurden und werden, die - unabhängig von ihrer funktionsgebundenen Wesenheit stets als autonome Werke aufgefasst und als solche bereits kurz nach ihren theatralen Uraufführungen auch im Konzertsaal aufgeführt wurden. Dass Stravinskij selbst einen großen Teil dazu beitrug, dass seine Ballette schon früh ohne Tanz (und Bühnenbild) aufgeführt wurden, scheint ein Teil der Wissenschaft als eine Art Absolution des Komponisten zu verstehen, die dazu berechtigt, die urspüngliche Konzeption als Ballett auszuklammern. ${ }^{83}$ Der Sacre wird von der Musikwissenschaft also sowohl als Ballett(musik) (das heißt

82 Für eine Erläuterung des Begriffs der Gebrauchsmusik vgl. Anm. 48.

83 Zum Ausklammern des Ballettursprungs des Sacre aus der wissenschaftlichen Betrachtung vgl. zum Beispiel van den Toorn, Stravinsky and ,The Rite of Spring', S. 1-3. Stravinskij selbst verleugnete die theatralen Wurzeln des Sacre erstmals in einem Interview mit Michel Georges-Michel, das 1920 in der französischen Zeitschrift Comøedia erschien, wenn er darin sagt: „Mais considérez bien que cette idée vient de la musique et non la musique de cette idée. J'ai écrit une œuvre architectonique et non anecdotique." Michel Georges-Michel: „Les Deux ,Sacre du printemps“', in: Comoedia, 11. Dezember 1920, S. 1. Das Interview ist auch abgedruckt bei François LÉSURE in Zusammenarbeit mit Gertraut Haberkamp, Malcom Turner und Emilia Zanetti (Hg.): Igor Stravinsky. ,Le Sacre du printemps: Dossier de presse (Anthologie de la Critique musicale. Dossiers de Presse, Bd. 1), Genf 1980, S. 53. Bei Truman Bullard findet sich ein Ausschnitt daraus. Vgl. Truman Campbell Bullard: The First Performance of Igor Stravinsky's, Sacre du Printemps', PhD Dissertation, 3 Bde., University of Rochester 1971, Bd. 1, S. 3. Stravinskij distanzierte sich in diesem Interview von Nižinskijs Choreografie, um die neue Sacre-Choreografie von Leonid Mjasin zu protegieren, die vier Tage vor Erscheinen des Interviews Premiere gefeiert hatte. Vgl. Joseph, Stravinsky's Ballets, S. 263. Nach 1920 bekräftigte Stravinskij immer wieder, dass es sich beim Sacre um ein rein musikalisches Werk handeln würde. Vgl. Taruskin, „Stravinsky and the Subhuman“, S. 37of. Unter anderem findet sich in seinen Expositions and Developments die Aussage: „I prefer ,Le Sacre' as concert piece.“ Igor Stravinsky und Robert CRAFt: Expositions and Developments, London 1962, S. $164 f$. 
mit Berücksichtigung seiner ursprünglichen Wesenheit als Bühnenwerk) als auch als autonome (Ballett-)Musik untersucht. Sowohl Bühnen- als auch autonomem Werk tritt man hierbei fast ausschließlich aus einer historiografischen und musikanalytischen Perspektive entgegen. Und so erweist sich der Untersuchungsgegenstand Sacre - vergleichbar mit der gesamten Ballettgattung bereits im innerdisziplinären musikwissenschaftlichen Diskurs als nicht ganz eindeutig bestimmbar. Aufgrund der vergleichbaren Herangehensweisen analytischer wie historiografischer Art werden diese unterschiedlichen Diskurse allerdings wechselseitig von den jeweiligen Vertretern wahrgenommen und gegebenenfalls auch kommentiert. So stellen beispielsweise beide Seiten sowohl den Ursprung im Ballett als auch die Nähe des Sujets zum Primitivismus nicht infrage. Van den Toorn akzeptiert zum Beispiel die Erkenntnisse Taruskins, obwohl deren zentrale Bestandteile - sowohl die russischen Wurzeln als auch Nähe des Ballettsujets zum Primitivismus - keine Auswirkungen auf seine Sacre-Analysen haben:

[...] the scenario itself, the choreography, and, above all, the close ,interdisciplinary conditions of coordination under which the music is now known to have been composed - these are matters which, after the 1913 premiere, quickly passed from consciousness. Like pieces of a scaffolding, they were abandoned in favor of the edifice itself and relegated to the ,extra-musical'. They became history, as opposed to living art. Even the title, with its clear suggestion of pagan rites or ,primitivism', lost its specific ties to the subject matter and became almost exclusively a musically descriptive label. ${ }^{84}$

Wie ändert sich nun das Bild, öffnet man den Blick über die musikwissenschaftlichen Grenzen hinaus und zieht Sacre-Untersuchungen aus dem Bereich der Tanzwissenschaft hinzu? Existiert auf interdisziplinärer Ebene ebenfalls ein gegenseitiges Wahrnehmen oder wechselseitiges Aufeinanderwirken, wie es sich im - wenn auch ungeeinten - musikwissenschaftlichen Diskurs zeigt? Und falls ja, welche Erkenntnisse können daraus bezüglich inner- wie interdisziplinär geführter Diskurse gewonnen werden?

\subsection{Innerdisziplinäre Sacre-Diskurse der Tanzwissenschaft}

Auch für die Tanzwissenschaft ist Stravinskijs Ballettwerk von besonderem Interesse - immerhin kooperierte er mit den bedeutendsten Choreografen des 20. Jahrhunderts, und sein musikalisches Schaffen war maßgeblich an der von Djagilev ausgehenden Modernisierung des Balletts beteiligt. ${ }^{85}$ Sowohl die

84 Van den Toorn, Stravinsky and,The Rite of Spring', S. 2.

85 Zunächst entstehen im Kontext der von Sergej Djagilev gegründeten Ballets Russes bedeutende Werke in Zusammenarbeit mit Michail Fokin (L'Oiseau de feu, Pétrouchka), 
deutsche als auch die englischsprachige Tanzwissenschaft versteht den Sacre dabei vorrangig als choreografisches Werk, das sich nicht nur in Nižinskijs Choreografie präsentiert, sondern auch in zahlreichen choreografischen Folgeversionen auf Stravinskijs Musik.

Nižinskijs Choreografie kam - nachdem der Uraufführungsskandal abgeklungen war - sehr lange nur wenig bis gar keine Aufmerksamkeit zu. Nach nur acht Aufführungen wurde der Sacre aus dem Repertoire der Ballets Russes genommen; weniger als ein Jahr später, im Februar 1914, wurde die dazugehörige Musik erstmals konzertant aufgeführt. Erst 1920 wurde das Werk ein weiteres Mal als Ballett produziert - nun allerdings nicht mehr mit Nižinskijs Choreografie, sondern in einer neuen choreografischen Version von Leonid Mjasin. ${ }^{86}$ Während Presse und Publikum Stravinskijs Partitur, aus dem Ballettkontext gelöst, schon kurz nach der Premiere zum Meisterwerk kürten, geriet Nižinskijs Choreografie mehr und mehr in Vergessenheit und ist daher nur bruchstückhaft überliefert. ${ }^{87}$ Der gefeierte Tänzer Nižinskij wurde nach seinem Ausscheiden aus den Ballets Russes 1913 Symbol des naiven und tragischen Genies, dem einflussreiche Personen immer wieder fehlendes Talent im Choreografieren nachsagten oder ein geringes Maß an Musikalität unterstellten. ${ }^{88}$ Nižinskijs Schizophrenie, die bereits wenige Jahre

Vaclav Nižinskij (Le Sacre du printemps) und Leonid Mjasin (Pulcinella). Mit Bronislava Nižinskaja (Les Noces) führt Stravinskij diese Bewegung weiter und wird schließlich mit George Balanchine (Apollon Musagète, Jeu de cartes, Orpheus, Agon) zum Mitbegründer des neoklassischen Balletts.

86 Die erste konzertante Aufführung des Sacre fand am 18. Februar 1914 in Sankt Petersburg unter der Leitung von Sergej Kusevickij (Serge Koussevitzky) statt. Vgl. Peter HiLL: Stravinsky. ,The Rite of Spring', Cambridge 2000, S. 8. Die prominentere konzertante Premiere in Paris unter Pierre Monteux datiert auf den 5. April 1914. Vgl. van den Toorn, Stravinsky and ,The Rite of Spring', S. 6.

87 Von Nižinskijs Choreografie sind lediglich Notationsskizzen, Bewegungszeichnungen (von Valentine Gross), Kostüm- und Bühnenbildgrafiken sowie mündliche Überlieferungen erhalten, vgl. Gabriele BrAndstetTER: „Le Sacre du printemps' 1913/2013“, in: Sacré 101. An Anthology on ,The Rite of Spring', hg. von Raphael GygAX, Zürich 2014, S. 149-161, hier S. 151. Die Bleistiftskizzen der damaligen Kunststudentin Valentine Gross, die diese während der Proben und Aufführungen in Paris anfertigte, sind Teil der Ekstrom Collection des Victoria \& Albert Museum, London, THM/7/8/3/1. Einige der Skizzen wurden bereits veröffentlicht, so unter anderem bei Richard BUCKLE (Hg.): Nijinsky on Stage. Action Drawings by Valentine Gross of Nijinsky and the Diaghilev Ballet Made in Paris between 1909 and 1913. Being a Donation to the Museum of Theatre Arts, London, by Jean Hugo. With a Chronology by Jean Hugo and an Introduction and Notes by Richard Buckle, London 1979, S. 131-141.

88 Auch auf Stravinskij ist es unter anderem zurückzuführen, dass Nižinskij lange Zeit choreografisches sowie musikalisches Können aberkannt wurden. Vgl. Hanna Järvinen: Dancing Genius. The Stardom of Vaslav Nijinsky, Basingstoke 2014, S. 15; Charles M. Joseph 
nach der Sacre-Premiere diagnostiziert wurde, erschwerte der Tanzwissenschaft wohl auch eine qualifizierte Auseinandersetzung mit ihm. ${ }^{89}$ Erst ab den 1970er-Jahren begann schließlich eine Neubewertung und damit einhergehende Aufwertung von Nižinskij und seiner Sacre-Choreografie. ${ }^{90}$ Heute sieht man in ihm einen der innovativsten Choreografen des 20. Jahrhunderts. ${ }^{91}$ Gabriele Brandstetter repräsentiert die gängige Sichtweise der internationalen Tanzwissenschaft, wenn sie Nižinskijs Sacre (analog zu Stravinskijs Sacre für die Musikwissenschaft) als „Choreographie der Grenzüberschreitung“92 von der Tradition in die Moderne beschreibt. Und genau hierin liegt wohl auch die Janusköpfigkeit der Choreografie begründet: Einerseits bildet Nižinskijs Sacre als historisches und zugleich verloren gegangenes Werk für die Tanzforschung den Höhepunkt einer jahrhundertealten Balletttradition, mit der Nižinskij brach. Andererseits avancierte der Sacre durch seine mittlerweile über 100 Jahre andauernde choreografische Rezeptionsgeschichte zu einem Schlüsselwerk des modernen Tanzes: Kein anderes Werk der Moderne wurde seit seiner Uraufführung in so zahlreichen Fassungen neu geschaffen. ${ }^{93}$

und Hannah Järvinen fassen die negativen Aussagen von Stravinskij und anderen über Nižinskijs Choreografie zusammen. Vgl. Joseph, Stravinsky's Ballets, S. 263; Järvinen, Dancing Genius, S. 11.

89 Vgl. Garafola, Diaghilev's Ballets Russes, S. 51f. Lynn Garafolas Äußerungen zu Nižinskij können als beispielhaft für den vorherrschenden Nižinskij-Diskurs gesehen werden. Hanna Järvinen vermutet hierin eine gewisse Vereinfachung und plädiert für eine Neubewertung der Ballets-Russes-Geschichte. Vgl. Järvinen, Dancing Genius, S. 1-26.

90 Vgl. Jordan, Stravinsky Dances, S. 413. Stephanie Jordan nennt als Initiator der Wiederbeschäftigung mit Nižinskijs Choreografie die beiden in den 197oer-Jahren über ihn erschienenen Publikationen von Richard Buckle und Lincoln Kirstein, auf die dann in den 198oer-Jahren der Sacre-Rekonstruktionsversuch von Millicent Hodson und Kenneth Archer folgte. Vgl. Richard BucKLE: Nijinsky, New York 1971; Lincoln Kirstein: Nijinsky Dancing, New York 1975; Millicent Ho Dson: Nijinsky's Crime against Grace. Reconstruction Score of the Original Choreography for, Le Sacre du printemps', New York 1996 sowie Anm. 94. Für ausführliche Angaben zur frühen und aktuellen Ballets-Russes-Geschichtsschreibung vgl. Järvinen, Dancing Genius, S. 6-15. Die Premiere des Rekonstruktionsversuchs erfolgte 1987 mit dem Joffrey Ballet in New York. 1989 wurde die Produktion aufgezeichnet. Vgl. Thomas Grimm und Kudy Kinberg (Regie und Produktion): The Search for Nijinsky's, Rite of Spring;, WNET/THIRTEEN und Danmarks Radio 1989.

91 Vgl. Jordan, Stravinsky Dances, S. 32.

92 Brandstetter und Klein, „Bewegung in Übertragung“, S. 17.

93 Einen Überblick über die weltweit entstandenen Sacre-Choreografien seit 1913 gibt die 2002 ins Leben gerufene Datenbank Stravinsky the Global Dancer der Roehampton University. Vgl. Stephanie Jordan und Larraine Nicholas: Stravinsky the Global Dancer. A Chronology of Choreography to the Music of Stravinsky, London Roehampton University, online verfügbar unter http://urweb.roehampton.ac.uk/stravinsky (Stand: September 2017). 
Was die tanzwissenschaftliche Beschäftigung mit Nižinskijs Sacre als historischem Gegenstand angeht, beschränkt sie sich größtenteils auf den englischsprachigen Raum. Hier entstanden ab den 198oer-Jahren grundlegende historiografische Studien von Millicent Hodson und Kenneth Archer sowohl zu Nižinskijs Choreografie als auch zu Nicholas Rerichs Bühnenbild und Ausstattung. Hierbei gewonnene Erkenntnisse zu Bewegungssprache und Kostüm- wie Bühnendesign flossen auch in den Rekonstruktionsversuch ein, den Hodson und Archer gemeinsam erarbeiteten und der erstmals 1987 auf der Bühne zu sehen war. ${ }^{94}$ Die amerikanische Tanzwissenschaftlerin

$94 \mathrm{Zu}$ diesem Rekonstruktionsversuch und den verwendeten Quellen vgl. Millicent Hodson: „The Fascination Continues. Searching for Nijinsky's ,Sacre“, in: Dance Magazine 54/6 (1980), S. 64-75; DIEs.: Nijinsky's New Dance. Rediscovery of Ritual Design in ,Le Sacre du Printemps', PhD Dissertation, University of California (Berkeley) 1985; DIEs.: „Nijinsky's Choreographic Method. Visual Sources from Roerich for ,Le Sacre du Printemps“', in: Dance Research Journal 18/2 (1986/1987), S. 7-15; DIES.: „Sacre'. Searching for Nijinsky's Chosen One“, in: Ballet Review 15/3 (1987), S. 53-66; dies., Nijinsky's Crime against Grace; DIES.: „Puzzles choréographiques. Reconstitution du ,Sacre‘ de Nijinsky“, in: ,Le Sacre du Printemps de Nijinsky, hg. von Etienne Souriau u.a., Paris 199o, S. 4574; Kenneth Archer: „Nicholas Roerich et la genèse du ,sacre“', in: ebd., S. $75^{-95}$. Mehrere Wissenschaftler und Tanzkritiker haben bereits darauf hingewiesen, dass eine Rekonstruktion von Nižinskijs Sacre aufgrund der Quellenlage nicht möglich sei - so zum Beispiel besonders prominent Joan Acocella im New Yorker: „(In 1987, an American dance scholar, Millicent Hodson, mounted a version of ,The Rite of Spring' on the Joffrey Ballet, but, from what one can tell, it was based on evidence too fragmentary to allow it to be counted as as actual reconstruction) [...] Who can say whether ,Le Sacre du Printemps' was in fact the great modernist masterpiece that it is now claimed to be? Perhaps it was something more like the shaggy, dolly, pseudo-folkloric thing that we saw at the Joffrey Ballet ,reconstruction'. Many of those who were dissappointed by the Joffrey version simply concluded that it's flatness was due to it's having been put together from such scrappy evidence - in other words, that it wasn't really Nijinsky. But who knows?" Joan ACOCELlA: „After the Ball was Over“, in: The New Yorker, 18. Mai 1992, Wiederabdruck in: Twenty-eight Artists and Two Saints. Essays, hg. von DIEs., New York 2007, S. 169-19o, hier S. 170-185. Für weitere kritische Stimmen vgl. unter anderem Järvinen, Dancing Genius, S. 16. Auch Millicent Hodson war sich der Problematik bewusst. Sie selbst gibt an, dass einige Teile der Originalchoreografie gar nicht oder nur äußerst ungenau überliefert sind. Vgl. Kenneth Archer und Millicent Hodson: „Confronting Oblivion. Keynote Adress and Lecture Demonstration on Reconstructing Ballets", in: Preservation Politics. Dance Revived. Reconstructed. Remade. Proceedings of the Conference at the University of Surrey Roehampton. November 1997, hg. von Stephanie Jordan, London 2000, S. 1-20, hier S. 2: „Before we [gemeint sind Millicent Hodson und der britische Kunsthistoriker Kenneth Archer] commit ourselves to work on a project, we do an evaluation of the types and amounts of information that we know survive or we think we can discover. We have to be convinced that the ultimate result will be based on at least $50 \%$ hardcore evidence both for dance and for design. The press and public always ask at the time of the premiere, ,What percentage of the original does the reconstruction represent?' For ,Le Sacre du 
Lynn Garafola bot in den 1980er-Jahren erstmals einen breiten Überblick über Kontext und Errungenschaften der Ballets Russes. Viele tanzwissenschaftliche Studien dazu fußen auch heute noch auf diesem frühen Forschungskanon. ${ }^{95}$ So stellt Stephanie Jordan ihrer wohl einmaligen musikchoreografischen Analyse von ,Nižinskijs' Sacre ${ }^{96}$ einen historiografischen Teil voran, der grosso modo auf dem bestehenden Kanon basiert. Als Grundlage ihrer Analyse wählt sie den von Hodson und Archer vorgelegten Rekonstruktionsversuch. ${ }^{97}$ Neuere Beiträge historiografischer Art zu den Ballets Russes im Allgemeinen und zu Nižinskij im Besonderen sind selten, finden sich aber unter anderem bei Tim Scholl oder Hanna Järvinen. ${ }^{98}$

Was die umfangreiche, zeitgenössische choreografische Auseinandersetzung mit dem Sacre angeht, so wird darunter in der Tanzforschung als ihrem wesentlichen Motor nicht selten die Wesenheit eines „verlorene[n] Ballett[s]" ${ }^{\text {“99 }}$ (Garafola) oder eine (unter anderem) durch den Verlust der Originalchoreografie hervorgerufene „Arbeit am Mythos“"100

printemps, our first reconstructed ballet, Joffrey [gemeint ist der US-amerikanische Tänzer und Choreograf Robert Joffrey] answered for us. He gauged $85 \%$. His response was based on the density of information we showed him in each area; choreography and performance style, costumes and decor, music and lighting. Density is the measure, and, if we agree ,Le Sacre du printemps' was $85 \%$, then we estimate ,Cotillon' was about $95 \%$, ,Till Eulenspiegel' and ,La Chatte' $75 \%$ and ,Skating Rink' and ,Jeux' $65 \%$.“

95 Järvinen kritisiert, dass in der Tanzgeschichtsschreibung allzu oft kanonisierte Argumente unreflektiert wiedergegeben würden. Viele dieser ,Fakten` - auch zu den Ballets Russes würden auf unwissenschaftlichen Studien basieren, die berichtigt werden müssten. Vgl. Järvinen, Dancing Genius, S. 7. Tatsächlich werden viele in den 1980er-Jahren erschienene prägende tanzwissenschaftliche Studien zu den Ballets Russes nur selten kritisch hinterfragt - so zum Beispiel Lynn Garafolas Diaghilev's Ballets Russes. Ihre umfangreiche und grundlegende Studie bietet damals wie heute einen wertvollen historischen Überblick über die Geschichte der Ballets Russes; sie enthält - sicherlich auch aufgrund der Komplexität des betrachteten Gegenstands - aber auch einige Lücken, die Fragen aufwerfen, derer sich bislang nur äußerst selten angenommen wurde.

96 Zur Vereinfachung soll der Sacre-Rekonstruktionsversuch von Millicent Hodson im Folgenden mitunter als ,Nižinskijs' Sacre bezeichnet werden, um ihn so vom Original (Nižinskijs Sacre) zu unterscheiden.

97 Stephanie Jordan ist sich der Problematik des Rekonstruktionsversuchs zwar bewusst, begründet die Auswahl jener Choreografie für ihre musikchoreografische Analyse des Sacre aber damit, dass die Rekonstruktion das Nächste sei, was ihr von Nižinskijs Sacre zur Verfügung stehe. Vgl. Jordan, Stravinsky Dances, S. 421.

98 Vgl. unter anderem Tim Scholl: From Petipa to Balanchine. Classical Revival and the Modernization of Ballet, London 1994; Järvinen, Dancing Genius.

99 Lynn Garafola: „A Century of Rites. The Making of an Avant-Garde Tradition“, in: Sacré 101, hg. von Gygax, S. 23-32, hier S. 25: „Precisely it is a lost ballet [...].“ Deutsche Übersetzung, ebd., S. 166.

100 Brandstetter und Klein, „Bewegung in Übertragung“, S. $17 f$. 
(Brandstetter) verstanden. ${ }^{101}$ Garafola verortet in diesem Vakuum eine gewisse konzeptionelle Freiheit, die eine choreografische Auseinandersetzung begünstigt und zeitgleich fortdauernde Impulse für eine Beschäftigung mit Ideen gibt, die dem Original nahestehen oder darin bereits angelegt zu sein scheinen. Als dem Ballett inhärent benennt sie dessen oszillierenden Zustand zwischen Primitivismus und Modernismus sowie dem Primitivismus unterzuordnende Topoi wie Biologismus, Opfer, Gewalt und Skandal. Als weitere zentrale Idee kennzeichnet sie die radikale Ablehnung der traditionellen Ballettästhetik. Beide markieren für sie einen Zustand der Überschreitung, der wiederum das Modell eines formalen Radikalismus für eine Umsetzung auf der Bühne bildet. ${ }^{102}$ Garafola ist davon überzeugt - in gewisser Weise verwandt zu Taruskins Verortung des von Stravinskij verwendeten musikalischen Materials in der russischen Tradition -, dass der Ursprung des Sacre'schen Primitivismus in der Balletttradition des 19. Jahrhunderts auszumachen ist. ${ }^{103}$

Brandstetter geht noch einen Schritt weiter: Da im Sacre für das 20. Jahrhundert zentrale ästhetische, ethnische und politische Themen aufscheinen würden, mit denen sich zeitgenössische Choreografen bis heute auseinandersetzten - sie möchte hiermit vor allem „die Frage nach der Rolle des Opfers, die eigentümliche Verschränkung von Ritualität, Primitivismus und Moderne und die ästhetische Debatte zu Ornament und Abstraktion" ${ }^{\prime 104}$ verhandelt wissen -, könne er in seinem Nicht-mehr-Vorhandensein bzw. Verlorensein als Meta-Choreografie und damit gleichsam Allegorie des modernen Tanzes verstanden werden. ${ }^{105}$ In ihrer „Re-Lektüre von Sacre $1913^{{ }^{406}}$ skizziert Brandstetter das Kulturmuster, das (ihr) als paradigmatisch für die Moderne gilt: nämlich den Diskurs von Wissenschaft und Kunst zu Anfang des

101 Die Tanzwissenschaftlerin Anette Hartmann stellt die Frage, ob der Reiz des Sacre nicht vielleicht auch in der Musik Stravinskijs bzw. in den durch die Musik hervorgerufenen körperlichen Reaktionen zu suchen sei, und benennt Choreografen, die die Musik als Impuls für ihre Auseinandersetzung mit dem Werk angeben. Vgl. Annette HaRTMANN: „Dancing Noises of Modern Life. ,Le Sacre du Printemps““, in: Strawinskys ,Motor Drive“ (Aesthetica Theatralia, Bd. 7), hg. von Monika Woitas und Annette Hartmann, München 2010, S. 211-227. Tatsächlich sollten die Bewegungen der Tänzer im Sacre durch die Rhythmen der Musik kontrolliert werden. Vgl. hierzu die Analyse in Kapitel III.1.3.

102 Vgl. Garafola, „A Century of Rites“, S. 25.

103 Vgl. Joan Acocella, Jonnie Greene und Lynn Garafola: „The Rite of Spring Considered as Nineteenth-Century-Ballet", in: Ballet Review 20/2 (1992), S. 68-71.

104 Brandstetter, „,Le Sacre du printemps“ 1913/2013“, S. 151.

105 Vgl. Brandstetter und Klein, „Bewegung in Übertragung“, S. 17 f.

106 Gabriele Brandstetter: „Ritual als Szene und Diskurs. Kunst und Wissenschaft um 1900 - am Beispiel von ,Le Sacre du printemps“', in: Konzepte der Moderne, hg. von Gerhard von Graevenitz, Stuttgart 1999, S. 367-388, hier S. 371. 
20. Jahrhunderts, der „die Erfindung des Primitiven [...] in Szene setzt: als Bild eines anderen Kulturmusters und zugleich in der Funktion einer Diagnose der eigenen Kultur ${ }^{407}$. Durch die Platzierung des historischen Sacre - also Nižinskijs Sacre von 1913 - in ebendiesem Kulturmuster legt sie Zusammenhänge frei, die seither von kulturwissenschaftlicher Seite als paradigmatisch angesehen werden. ${ }^{108}$ Ein großer Teil der deutschsprachigen tanzwissenschaftlichen Betrachtungen zum Sacre ist genau in dieser kulturwissenschaftlich orientierten Lesart zu verstehen: den Themen Opfer, Ritualität, Primitivismus, Modernismus, Ornament und Abstraktion gehen sie weniger aus einer historischen Perspektive nach; vielmehr dienen ihnen diese dem Ballett (scheinbar) eingeschriebenen Motive dazu, aktuelle Herangehensweisen zeitgenössischerChoreografen aus kulturwissenschaftlicherSichtzu beleuchten. ${ }^{109}$

So divers die inner- wie interdisziplinären Diskussionen zum Ballett im Allgemeinen und zum Sacre im Besonderen zunächst wirken mögen, in einem Punkt scheinen Musik- und Tanzwissenschaft einig: Das Oszillieren des Sacre zwischen Primitivismus und Modernismus stellt sich prima vista als ein mal mehr, mal weniger ausgeprägt - alle Diskurse einender Gedanke dar. Im Folgenden soll daher erprobt werden, ob jene musik- und tanzwissenschaftlichen Untersuchungen, die unter dem Begriff Primitivismus zu verorten sind, bereits als zwei unterschiedliche Ausprägungen eines von Tanz- und Musikwissenschaft gemeinsam geführten Diskurses verstanden werden können.

\subsection{Der Primitivismusdiskurs als Gemeinsamkeit von musik- und tanzwissenschaftlicher Sacre-Forschung?}

William Rubin, der amerikanische Kunsthistoriker und langjährige Kurator des Museum of Modern Art, beschrieb 1984 im für die Kunstgeschichte zentralen Ausstellungskatalog Primitivism in Twentieth Century Art den Begriff Primitivismus als eine europäische Kunstrichtung, die sich von Artefakten

107 Ebd.

108 Vgl. ebd., S. 371-374.

109 Unter anderem folgende Texte zum Sacre sind in diesen kulturwissenschaftlichen Kontext einzuordnen: Gabriele Brandstetter und Gerhard Neumann: „Opferfest. ,Penthesilea' - ,Sacre du Printemps““, in: Konflikt. Grenze. Dialog. Kulturkontrastive und interdisziplinäre Textzugänge. Festschrift für Horst Turk zum 6o. Geburtstag, hg. von Jürgen Lehmann u.a., Frankfurt a.M. u.a. 1997, S. 105-139; Brandstetter, „Ritual als Szene und Diskurs“; Katherina ZAKRAVskY: „Re-Membering ,le Sacre““, in: Tanz anderswo. Intraund Interkulturell (Jahrbuch Tanzforschung, Bd. 14), hg. von Krassimira Kruschкova und Nele LipP unter Mitarbeit von Sabine Karoß, Münster 2004, S. 205-235; Alexander Schwan: „Queer Stupidity. Ornament und Primitivismus in ,Le Sacre du Printemps“", in: Primitivismus intermedial (Colloquium Helveticum 44 [2015]), hg. von Nicola Gess, Christian Moser und Markus WinkLer, Bielefeld 2015, S. 69-89. 
westafrikanischer und ozeanischer Stammeskulturen und deren Gestaltung anregen ließ, und startete damit eine wissenschaftliche Debatte zum Phänomen des Primitivismus in der bildenden Kunst, die sich mittlerweile auch in andere wissenschaftliche Disziplinen ausgeweitet hat, die vergleichbare Phänomene in Literatur, Tanz oder Musik untersuchen. ${ }^{110}$ Dass ein solches, eher eng gefasstes Primitivimusverständnis nicht ohne Weiteres auf andere Künste übertragbar ist, darauf machte für die Literatur bereits 2005 Erhard Schüttpelz aufmerksam, indem er feststellte, dass der „Augenfälligkeit der Vorbild-Beziehung "111, die den Primitivismus im Bereich der bildenden Kunst auszeichne, im Bereich des Sprachlichen die Sprache selbst „widersprach und widerspricht ${ }^{\text {"112 }}$. Ein semantisch wie historisch erweitertes Primitivismusverständnis, wie es sich in der Kunstgeschichte auf Grundlage des von Rubin vertretenen enger gefassten Begriffs im Laufe der Zeit entwickelte, erleichtert es der Literaturwissenschaft allerdings, analoge Entwicklungen in der Literatur zu untersuchen. Dieser erweiterte Begriff bezeichnet als ,primitiv' nicht nur westafrikanische und ozeanische Stammeskunst, sondern außerdem auch andere außereuropäische Kunst, Volkskunst, Kunst des Mittelalters oder Kunst von Kindern und psychisch kranken Menschen. Außerdem verweist er darauf, dass das Interesse an primitiver Kunst Anfang des 20. Jahrhunderts Bestandteil eines allgemeinen Interesses an primitiven Kulturen darstellte. ${ }^{113}$ Auf solch einem erweiterten Verständnis des ,Primitiven fußend, vermuten die Herausgeber des bereits erwähnten Sammelbands Primitivismus intermedial ${ }^{114}$, dass sich der „Primitivismus der Moderne von Anfang an in einem Feld intensiven intermedialen Austauschs ${ }^{\text {“115 }}$ entwickelte.

In musikwissenschaftlichen Schriften zur Moderne sind der Begriff Primitivismus sowie ihm verwandte Termini wie Archaismus, Archaik, Archaisches oder Ursprüngliches weit weniger geläufig als in vergleichbaren Studien der Kunst- oder Literaturwissenschaft. Als Stichwörter in Musiklexika oder Einträge in musikwissenschaftlichen Handbüchern sind sie nur

110 Vgl. Nicola Gess: „Literarischer Primitivismus. Chancen und Grenzen eines Begriffs“, in: Literarischer Primitivismus, hg. von DIEs., Berlin 2013, S. 1-9; DIEs., Christian Mose R und Markus Winkler: „Vorwort“, in: Primitivismus intermedial, hg. von dies., S. 9-14, hier S. 8f. Zum Primitivismusbegriff von William Rubin vgl. William Rubin: „Modernist Primitivism. An Introduction“, in: ,Primitivism 'in 2oth Century Art. Affinity of the Tribal and the Modern, 2 Bde., hg. von DERs., New York 1984, Bd. 1, S. 1-84.

111 Erhard Sс нӥтtр LL: Die Moderne im Spiegel des Primitiven. Weltliteratur und Ethnologie (1870-1960), München 2005, S. 36o.

112 Ebd.

113 Vgl. Gess, „Literarischer Primitivismus. Chancen und Grenzen eines Begriffs“, S. 2f.

114 Vgl. Anm. 137.

115 Gess, Moser und Winkler, „Vorwort“, S. 10. 
sporadisch bis gar nicht zu finden, ${ }^{116}$ und so verwundert es nicht, dass die Frage, welche musikalischen Strömungen des frühen 20. Jahrhunderts mit Primitivismus wohl am treffendsten benannt werden könnten, bislang als ungeklärt gilt ${ }^{117}$ - mit Ausnahme von Stravinskijs Sacre. Denn wenn der Begriff im musikbezogenen Kontext doch einmal fällt, dann gemeinhin mit Verweis auf ebendieses Ballett. ${ }^{118}$ Da eine zu der von Schüttpelz für die Literatur vorgelegten folgenreichen Untersuchung vergleichbare kulturwissenschaftlich orientierte Studie für die Musikwissenschaft bislang nicht vorhanden ist, würde es schlichtweg zu weit gehen, von einem existenten musikwissenschaftlichen Primitivismusdiskurs zu sprechen. ${ }^{119}$ Was den direkten Transfer

116 Vgl. Gernot Gruber: „Das ,Archaische“ in der Musikkultur der Wiener Moderne. Eine Skizze“, in: Kunst. Kontext. Kultur. Manfred Wagner. 38 Jahre Kultur- und Geistesgeschichte an der Angewandten, hg. von Gloria Withalm, Anna Spонn und Gerald Bast, Wien 2012, S. 132-146, hier S. 132; Glenn WATKIns: Pyramids at the Louvre. Music, Culture, and Collage from Stravinsky to the Postmodernists, Cambridge (Mass.) 1994, S. 64. In den beiden Referenzlexika der Musikwissenschaft, der MGG und dem New Grove, gibt es keinen Eintrag zum Primitivismus. Auch einschlägige Handbücher zur Musik des 2o. Jahrhunderts sparen einen diesbezüglichen Vermerk aus. Vgl. unter anderem Danuser, Die Musik des 20. Jahrhunderts; Siegfried MaUser und Matthias Schmidt (Hg.): Geschichte der Musik im 20. Jahrhundert. 1900-1925 (Handbuch der Musik im 20. Jahrhundert, 14 Bde., wiss. Beirat: Elmar BUDDE u.a., Laaber seit 1999, Bd. 1), Laaber 2005.

117 Vgl. Stefan Weiss: „Vom ,russischen' zum ,sowjetischen Primitivismus‘. Wandlungen einer musikästhetischen Denkfigur im ersten Drittel des 2o. Jahrhunderts", in: Primitivismus intermedial, hg. von Gess, Moser und Winkler, S. 169-181, hier S. 169.

118 Dass Stravinskijs Sacre einem (musikalischen) Primitivismus zuzuordnen sei, findet sich in unterschiedlichen Texten immer wieder. So zum Beispiel bei Joseph AUnER: Music in the Twentieth and Twenty-First Centuries. Western Music in Context, New York u.a. 2013, S. 70-77: „Stravinsky's Rite of Spring [...] more than any other, established the link between Modernism, Primitivism, and the folk." Ebd., S. 70. Deborah Mawer wählt in ihrem Aufsatz über "Spiritual ,Otherness' in [André Jolivets] ,Mana“" den Sacre als Beispiel für einen musikalischen Primitivismus, wenn sie im ersten Abschnitt schreibt: „Jolivet shared the long established fascination with the exotic catalyzed by the Exposition universelle of 1889 and reinforced by the Exposition coloniale of 1931. His emergent practise is [...] connected to the primitivism of Igor Stravinsky's ,Sacre du printemps' (1913) [...]." Deborah Mawe R: „Jolivet's Search for a New French Voice. Spiritual ,Otherness in "Mana' (1935)“, in: French Music, Culture, and National Identity, 1870-1939, hg. von Barbara L. KeLLY, Rochester 2008, S. 172-193, hier S. 172. Gurminder Kaur Bhogal widmet dem Ornament und Neoprimitivismus im Sacre ein ganzes Kapitel. Vgl. Gurminder Kaur Bhogal: Details of Consequence. Ornament, Music, and Art in Paris, New York 2013, S. 212267. Taruskin formulierte hierzu 1997 Folgendes: „Primitivism is often touted, especially in discussions of ,The Rite of Spring', as one of those twentieth-century revolutions that cut our age off from the past [...]." Taruskin, „Stravinsky and the Subhuman“, S. 378.

119 Melanie Unseld weist in dem 2013 erschienenen Sammelband Historische Musikwissenschaft. Grundlagen und Perspektiven darauf hin, dass sich die Musikwissenschaft mit kulturwissenschaftlichen Perspektiven schwertue und an kulturwissenschaftlichen 
des im kunstgeschichtlichen Kontext verwendeten Primitivismusbegriffs auf den Bereich der Musik anbelangt, stellt der österreichische Musikwissenschaftler Gernot Gruber nachvollziehbar fest, dass dem erklingenden Medium Musik „die Unmittelbarkeit [der] Eindrücke von materiellen Kunstgegenständen über Jahrtausende hinweg [...] verwehrt bleibt“. ${ }^{120}$ Die einzige Möglichkeit, Musik und Tanz fremder ozeanischer wie afrikanischer Kulturen zu rezipieren, boten zur Jahrhundertwende die in Paris, London und Wien stattfindenden Weltausstellungen sowie die dort präsentierten exotischen Tanz- und Musikgruppen. ${ }^{121}$ Gruber lokalisiert die Suche eines Musikers oder Komponisten nach dem Archaischen ${ }^{122}$ daher weniger in den genannten ozeanischen wie afrikanischen Vorbildern, sondern vielmehr in "breit gestreuten Überlegungen zur Frage nach den Anfängen der Musik ${ }^{a 123}$. Die Ausführungen des amerikanischen Musikwissenschaftlers Glenn Watkins zu einem musikalischen Primitivismus weisen in eine vergleichbare Richtung. Neben der Faszination für afrikanische und ozeanische Vorbilder sieht er eine allgemeine Suche nach dem Ursprung der Menschheit bzw. der eigenen kulturellen Identität als ausschlaggebend für die Hinwendung zu ,primitiven` Artefakten. Da für den Bereich der Musik allerdings keine physischen Artefakte auszumachen waren, vermutet er, dass man sich derjenigen Zeugnisse bediente, in denen man die eigene kulturelle Identität am authentischsten eingeschrieben wähnte, und verweist auf Sammlungen nationaler Volksmusik, die Mitte bis Ende des 19. Jahrhunderts an nahezu allen (west-)europäischen Orten entstanden, sowie auf allgemeine Studien zu einer ruralen Ästhetik. ${ }^{124}$

Theoriediskussionen bislang nur verhalten teilnehme. Gleichzeitig merkt sie an, dass die Musikwissenschaft in den methodisch-theoretischen Diskussionen der Kulturwissenschaft größtenteils außen vor gelassen werde, obwohl das Phänomen der Musik darin meist ergiebig untersucht werde. Vgl. Melanie UnSELD: „Die Kulturwissenschaften als Herausforderung für die Musikwissenschaft - und was sich daraus für die Historische Musikwissenschaft ergibt", in: Historische Musikwissenschaft. Grundlagen und Perspektiven, hg. von Calella und Urbanek, S. 266-288, hier S. 267.

120 Gernot Gruber: „Webern und die Suche der Wiener Moderne nach dem ,Archaischen in der Musik“, in: Der junge Webern. Künstlerische Orientierungen in Wien nach 1900 (Webern-Studien. Beihefte der Anton-Webern Gesamtausgabe, Bd. 2a), hg. von Monika KRÖPfL und Simon ОвеRт, Wien 2015, S. 77-96, hier S. 79.

121 Vgl. ebd., S. 82. Vgl. in diesem Kontext auch die Studie von Annegret Fauser: Musical Encounters at the 1889 Paris World's Fair, Rochester 2005, S. 139-215.

122 Gruber verwendet hier den Begriff des Archaischen gleichbedeutend mit einem „positiv verstandenen Primitivismus“, vgl. Gruber, „Webern und die Suche der Wiener Moderne nach dem ,Archaischen“', S. 79 .

123 Ebd. Zu den verschiedentlichen Ursprungssuchen vgl. ebenfalls seine Ausführungen in ebd., S. $79-88$.

124 Vgl. Watkins, Pyramids at the Louvre, S. 7o. 
Der deutsche Musikwissenschaftler Stefan Weiss betont in seinem Aufsatz „Vom ,russischen' zum ,sowjetischen Primitivismus“', dass für den Bereich der Musik neben afrikanischen und afroamerikanischen Vorbildern das Russische bzw. Zentralasiatische als mindestens ebenso einflussreich anzusehen sei, und begründet dies damit, dass das Zurückgreifen auf Volksliedhaftes in Russland bereits als unabdingbare Voraussetzung einer gelungenen nationalen Komposition gegolten habe, „lange bevor man vom Primitivismus im eigentlichen Sinne sprechen konnte ${ }^{425}$. Das Sammeln und Studieren von Volksliedern sowie ein diesbezügliches Zitieren oder Imitieren sei bereits Ende des 19. Jahrhunderts eine gängige Praxis unter russischen Komponisten gewesen und - anders als in westeuropäischen Ländern - mehr als Qualitätsmerkmal denn als inferiorer Ausdrucksmodus gesehen worden. ${ }^{26}$ In all diesen Texten wird das Phänomen also primär als ein produktionsimmanenter Vorgang beschrieben, der im Falle Russlands sogar in der landeseigenen Musiktradition zu verankern sei. Wenn nun also Taruskins Erkenntnisse zum Sacre im musikwissenschaftlichen Kontext in Zusammenhang mit einem musikalischen Primitivismus gebracht werden, ${ }^{127}$ ist dies höchstwahrscheinlich darauf zurückzuführen, dass Taruskin im Sacre einen Rückgriff auf altes bzw. volkstümliches Material feststellte.

Auch in der Tanzwissenschaft scheint ein mit der Kunst- oder Literaturwissenschaft vergleichbarer Primitivismusdiskurs noch nicht etabliert. Möglicherweise aber aufgrund der Tatsache, dass sich die (deutschsprachige) Tanzwissenschaft aus der Literaturwissenschaft bzw. der Literaturwissenschaft verwandten Theaterwissenschaft entwickelte, zeigen sich hier Begriffsübertragungen als unproblematischer als innerhalb der Musikwissenschaft.

125 Weiss, „Vom ,russischen' zum , sowjetischen Primitivismus“", S. 16gf.

126 Vgl. ebd., S. 170. Über die Volksliedsammlungen des 19. Jahrhunderts in Russland und ihre Bedeutung für die dortige kompositorische Praxis vgl. Dorothea RedePenning: Geschichte der russischen und sowjetischen Musik, 2 Bde., Laaber 1994-2008, Bd. 1: Das 19. Jahrhundert, Laaber 1994, S. 303-312; vgl. DIES.: „Russischer Stoff, europäische Form. Der Dialog der Kulturen in der Musik“, in: Osteuropa 53/9f. (2003), S. 1262-1280. Zur ,nationalen Selbstfindung" und zur Sonderrolle des russisch-slawischen Ostens Europas im Umgang mit Volksliedmaterial im 19. Jahrhundert vgl. unter anderem Wolfram STE INBECK: „Musik und nationale Identifikation im 19. Jahrhundert“, in: Klang - Ton - Musik. Theorien und Modelle (national)kultureller Identitätsstiftung (Sonderheft 13 der Zeitschrift für Ästhetik und Allgemeine Kunstwissenschaft), hg. von Wolf Gerhard Sc нмidT, Jean-François CANDoni und Stéphane PeSnel, Hamburg 2014, S. 229-242. Über das Phänomen des nationalen Tons in der Musik vgl. unter anderem Siegfried OechSLE: „Nationalidee und große Symphonie. Mit einem Exkurs zum ,Ton“, in: Deutsche Meister - böse Geister? Nationale Selbstfindung in der Musik, hg. von Hermann DANUSER und Herfried MüNKLER, Schliengen 2001, S. 166-184.

127 Vgl. Kapitel I.2.1. 
Anders als in der Musikwissenschaft sind Primitivismus und unter ihm zu subsumierende Termini wie Ritualität, Opfer, Gewalt, Ornament oder Biologismus in der Tanzwissenschaft durchaus gebräuchlich. Den Sacre beschreibt Brandstetter beispielsweise in ihrem 1999 erschienenen Aufsatz „Ritual als Szene und Diskurs. Kunst und Wissenschaft um 1900 - am Beispiel von ,Le Sacre du printemps “ " als eine Darstellung bzw. Inszenierung eines fikitiven archaischen Rituals und fasst das Libretto folgendermaßen zusammen: „Das Thema des Balletts ist der Opfertod eines jungen Mädchens, das aus der Reihe von Jungfrauen in einem rituellen Prozeß auserwählt und zum Opfer stigmatisiert wird und sich schließlich zu Tode tanzt.“128 Ritual, Opfer und Tod gelten für Brandstetter als dem Sacre eingeschrieben. Was das Ritual angeht, so erkennt sie ein solches auch auf der Rezeptionsebene: Bezogen auf Studien des kanadischen Soziologen Erving Goffman und ausgehend von den Skandalberichten zur Uraufführung beschreibt sie das Zuschauer-Darsteller-Verhältnis um 1913 als ein soziales Ritual, das widerum durch bestimmte Konventionen und Regeln gekennzeichnet ist. Mit genau diesen Regeln sei am Premierenabend gebrochen worden. ${ }^{129}$ Auch den Begriff des Primitiven führt die Tanzwissenschaftlerin als ein rezeptionsgelenktes Phänomen ein: Nach einem kurzen Exkurs zum Primitivismusverständnis des Archäologen und Sacre-Librettisten bzw. -Bühnenbildners Nikolaj Rerich, der damit ein "Schlagwort der Zeit“130 aufgegriffen habe, beschreibt sie Wörter wie das Primitive, Wilde und Barbarische als dem Sacre vielfach ex post zugeschriebene Epitheta, die sie mit der Angabe von zeitgenössischen Rezensionsauszügen konkretisiert. ${ }^{131}$ Mit einem

128 Brandstetter, „Ritual als Szene und Diskurs“, S. 369.

129 Vgl. ebd., S. 368. Zu den von Brandstetter gesichteten Berichten zur Uraufführung vgl. ebd., Anm. 2. Die Konventionen und Regeln, mit denen am Premierenabend gebrochen wurde, bezeichnet sie in Anlehnung an Erving Goffman als „Rahmen-Ritual“. Vgl. ebd. sowie Erving Goffmann: Rahmen-Analyse. Ein Versuch über die Organisation von Alltagserfahrungen, Frankfurt a.M. 1980, S. 143-176; DERs.: Interaction Ritual. Essays in Face-to-Face Behavior, Chicago 1967.

130 Brandstetter, „Ritual als Szene und Diskurs“, S. 37o. Brandstetter macht in diesem Kontext darauf aufmerksam, dass das Thema des Primitiven bzw. des ,Barbaren' nicht nur zu Beginn der Moderne, sondern auch Ende des 2o. Jahrhunderts noch rege diskutiert wurde und verweist auf das 1996 erschienene soziologische Sammelwerk Modernität und Barbarei [...] am Ende des 20.Jahrhunderts. Vgl. Max Miller und Hans-Georg Soefffner (Hg.): Modernität und Barbarei. Soziologische Zeitdiagnose am Ende des 20.Jahrhunderts, Frankfurt a.M. 1996.

131 Vgl. Brandstetter, „Ritual als Szene und Diskurs“, S. 37o. Sie bestärkt ihre Aussage noch zusätzlich mit der Angabe, dass schon andere Tanzwissenschaftler den Sacre in die Nähe des Primitivismus gerückt hätten. Vgl. ebd. Als Beispiel führt sie einen Aufsatz der amerikanischen Tanzwissenschaftlerin und -kritikerin Joan Acocella an. Vgl. Joan Acocella: „Vaslav Nijinsky“, in: The Art of Enchantment. Diaghilev's Ballets Russes 
Hinweis auf die 1996 erschienene Kulturphilosophie des deutschen Philosophen Ralf Konersmann schildert Brandstetter das Primitive und Barbarische schließlich als Elemente einer Rhetorik der Krise ${ }^{132}$ und erkennt in ihnen ein für die Moderne paradigmatisches Kulturmuster, in dessen Kontext sie die Sacre-Choreografie situiert. ${ }^{133}$

Brandstetter verortet hier den Sacre'schen Primitivismus also primär auf der Ebene der Rezeption und beruft sich bei der Begriffsverwendung auf unterschiedliche Methoden der Soziologie, Philosophie und Literaturwissenschaft ${ }^{134}$ bzw. auf eine Begriffsverwendung zuvor erschienener tanzwissenschaftlicher Sacre-Beiträge. Eine theoretische Reflexion darüber, wie Primitivismus im spezifisch tanzwissenschaftlichen Kontext zu gebrauchen sei und wie Gruber, Watkins oder Weiss eine solche für die Musikwissenschaft versuchen, legt Brandstetter also nicht vor.

Bei den Tanzwissenschaftlerinnen Joan Acocella, Lynn Garafola und Jonnie Greene ist Ähnliches festzustellen. In ihrem Aufsatz „The Rite of Spring considered as Nineteenth-Century Ballet" von 1992 fällt der Primitivismusbegriff schon im zweiten Abschnitt, wenn sie auf die dem Sacre inhärenten Ideen verweisen, die ihrer Ansicht nach einen Großteil seines Faszinosums ausmachen. Für sie gilt (anders als für Brandstetter): „Of these ideas [gemeint sind all jene Ideen, die dem Sacre als eingeschrieben gelten] the most important is primitivism " 135 - Primitivismus als "the belief that society does not elevate or improve the human soul, but, on the contrary, corrupts it and that it is those things that are least socialized, least civilized - children, ,savages', raw emotion, plain speech - that are closest to truth “136. Die drei Forscherinnen verwenden den Terminus nicht wie Brandstetter als ein rein rezeptionsgelenktes Phänomen, nutzen ihn aber analog zu ihr für ihre tanzwissenschaftlichen Untersuchungen, ohne ihn zuvor einer fachimmanenten theoretischen Reflexion unterzogen zu haben. Auch wenn sie keine bestimmte

1909-1929, hg. von Nancy VAN Norman BAeR, San Francisco 1988, S. 96-111: „Sacre is one of the purest examples of primitivism."

132 Vgl. Brandstetter, „Ritual als Szene und Diskurs“, S. 369-371; Ralf Konersmann (Hg.): Kulturphilosophie, Leipzig 1996.

133 Vgl. Brandstetter, „Ritual als Szene und Diskurs“, S. 37of.

134 Brandstetter weist darauf hin, dass im Bereich der Literatur jene Hinwendung zum Fremden bzw. Anderen Anfang des 20. Jahrhunderts schon hinlänglich erforscht sei, und nennt entsprechende Beispiele. Vgl. ebd., S. 371.

135 Acocella; Garafola und Greene, „,The Rite of Spring“ Considered as Nineteenth-CenturyBallet", S. 68.

136 Ebd. 
Referenz nennen, so erinnert ihre Begriffsdefinition stark an jenes erweiterte Begriffsverständnis, welches von der Kunstwissenschaft nach Rubin sowie von aktuellen literaturwissenschaftlichen Theorien vorgeschlagen wird.

Während also in den betrachteten musikwissenschaftlichen Texten ein produktionsimmanentes Primitivismusverständnis festgestellt werden kann, weisen die untersuchten tanzwissenschaftlichen Texte in der Tendenz auf ein rezeptionsgelenktes Begriffsverständnis hin. Eine befriedigende Antwort auf die Frage, ob die unter Primitivismus zu verortenden Untersuchungen von Musik- und Tanzwissenschaft schon als ein gemeinsam geführter Diskurs zwischen Musik- und Tanzwissenschaft verstanden werden können, liefern die Texte allerdings nicht. Exemplarisch soll daher ein Blick auf den bereits erwähnten 2015 erschienenen Sammelband Primitivismus intermedial geworfen werden, der sich dem Primitivismusphänomen zu Beginn des 20. Jahrhunderts aus einer intermedialen Perspektive nähert und insbesondere sowohl einen tanz- als auch einen musikwissenschaftlichen Beitrag zum Sacre enthält. ${ }^{137}$

Stefan Weiss' musikwissenschaftlicher Aufsatz „Vom ,russischen“ zum ,sowjetischen Primitivismus “ beginnt nach einem kurzen Überblick zum Primitivismusverständnis der Musikwissenschaft ebenfalls auf der Ebene der Rezeption: Mit einem Zitat aus Stravinskijs 1935/36 erschienenen Chroniques de ma vie, in dem der Komponist ein Kindheitserlebnis schildert, macht Weiss darauf aufmerksam, wie sehr sich Stravinskij in den 193oer-Jahren zu einem Künstler stilisierte, „dessen Erweckungserlebnis durch die Begegnung mit dem musikalisch Undomestizierten erfolgte ${ }^{\text {“138}}$. Stravinskij schreibt:

Un paysan énorme assis sur le bout d'un tronc d'arbre. Une odeur pénétrante de résine et de bois coupé flatte les narines. Le paysan n'est vêtu que d'une courte chemise rouge. Ses jambes aux poils roux sont nues, aux pieds il a des sandales d'écorce. Sur sa tête, une forte chevelure, épaisse et rousse comme sa barbe, pas un cheveu blanc, - et c'était un vieillard. Il était muet, mais claquait très bruyamment de la langue et les enfants avaient peur de lui. Moi aussi. Pourtant la curiosité prenait le dessus. On s'approchait de lui, et alors, pour amuser les enfants, il se mettait à chanter. Ce chant - c'était deux syllabes, les seules qu'il

137 Unter dem Titel Primitivismus intermedial vereinen Nicola Gess, Christian Moser und Markus Winkler aktuelle, aus unterschiedlichen Fachdisziplinen stammende Texte zum Primitivismus. Dass in dieser Sammlung sowohl der tanz- als auch der musikwissenschaftliche Beitrag den Sacre als Beispiel heranziehen, kann als exemplarisch dafür gesehen werden, wie nahe Primitivismus und Sacre in beiden Fachdisziplinen gedacht werden. Vgl. Schwan, „Queer Stupidity“; Weiss, „Vom ,russischen“ zum ,sowjetischen Primitivismus'“. Hier bilden sie daher den Ausgangspunkt für eine allgemeine Reflexion über den Gebrauch des Primitivismusbegriffs von Musik- und Tanzwissenschaft.

138 Weiss, „Vom ,russischen` zum ,sowjetischen Primitivismus “', S. 171. 
pouvait prononcer, dénuées de tout sens, mais qu'il faisait alterner avec une dextérité incroyable dans un mouvement très vif. Il accompagnait ce gloussement de la façon suivante: il collait la paume de sa main droite sous l'aisselle gauche, puis, d'un geste rapide, faisait mouvoir le bras gauche en l'appuyant sur la main droite. Il faisait ainsi sortir de sous sa chemise une suite de sons assez suspects, mais bien rhythmés et que par euphémisme on pouvait qualifier de ,baisers de nourricé. Cela m'amusait follement et, à la maison, je me mettais à imiter cette musique avec beaucoup de zèle. Tant et si bien qu'on me défendit de me servir d'un accompagnement aussi indécent. ${ }^{139}$

Um das Hervorheben des Ruralen in Stravinskijs Aussage zu verorten, findet Weiss zwei Erklärungen: Zum einen vermutet er, dass Stravinskij damit auf einen positiv konnotierten Primitivismus Bezug nimmt und meint damit jenen von Brandstetter als Paradigma der Moderne beschriebenen Primitivismus, der dem Sacre vielfach ex post - also auf der Ebene der Rezeption zugeschrieben worden ist. Zum anderen erkennt er in Stravinskijs Wortwahl ein Anknüpfen an mehrere Generationen russischer Komponisten, die ihre maßgebliche Inspiration aus der ureigenen Volkskultur zogen, und rekurriert damit wiederum auf jenen produktionsimmanenten Primitivismusbegriff von Gruber und Watkins. Der rezeptionsgelenkte Primitivismusbegriff gibt ihm dann allerdings Anlass zur Frage nach dem Ursprung ebendieser im Kontext des Sacre hervorstechenden Konnotation des Primitiven durch das westeuropäische Publikum. Seine Reflexion über eine Denkfigur, welche die westeuropäische Rezeption russischer bzw. sowjetischer Musik schon vor, aber auch nach dem Sacre wesentlich bestimmte, schließt daran an. ${ }^{140}$

Auch der Tanzwissenschaftler Alexander Schwan versteht in seinem im selben Band publizierten Aufsatz „Queer Stupidity. Ornament und Primitivismus in Le Sacre du Printemps“ den Sacre'schen Primitivismus als ein rezeptionsgelenktes Phänomen. Als Ausgangspunkt wählt er den berühmten Sacre-Essay des französischen Schriftstellers Jacques Rivières, den dieser im November 1913 in der Nouvelle Revue Française veröffentlichte, ${ }^{141}$ und beschreibt, wie exzessiv die zeitgenössische Presse eine Äußerung Nižinskijs aufgriff, die dieser im Anschluss an die Londoner Sacre-Aufführungen (am 14. Juli 1913) in einem Interview in der Daily Mail getätigt hatte. Mit dem

139 Igor Strawinsky: Chroniques de ma vie, 2 Bde., Paris 1935, Bd. 1, S. $10 f$.

140 Vgl. Weiss, „Vom ,russischen' zum, sowjetischen Primitivismus“".

141 Vgl. Schwan, „Queer Stupidity“, S. 69-89; Jacques Riviè RE: „Le Sacre du Printemps““, in: La Nouvelle Revue Française 5/59, 1. November 1913, S. 705-730. Wiederabdruck in: Dossier de presse, hg. von Lésure, S. 38-48. 
Bekenntnis „I eat my meat without sauce Bérnaise“142 habe Nižinskij „,in das Herz des Primitivismus-Diskurses vor Beginn des ersten Weltkriegs ${ }^{\text {"143 }}$ getroffen und damit womöglich gleichermaßen Rivière zu dessen Essay inspiriert ${ }^{144}$ - jenem „ausführlichen Kommentar zu Le Sacre du printemps, dessen Reflexionen über das Verhältnis von Tanz, Modernismus und die Denkfigur des ,Primitiven

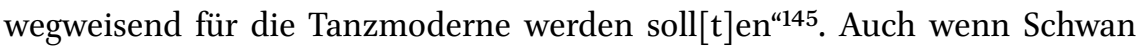
dies nicht explizit vermerkt: Seine Ausführungen weisen darauf hin, dass Rivières Text nicht nur die Tanzmoderne, sondern auch die tanzwissenschaftliche Sacre-Forschung beeinflusst hat. So zieht er zum Beispiel aus Rivières Beobachtungen von 1913 Rückschlüsse auf Nižinskijs Choreografie, um davon ausgehend Folgerungen für seine Thesen zu Primitivismus und Ornament im Sacre abzuleiten. ${ }^{146}$ Oder er wählt Rivières Angaben als Referenz, um die ruckhaften Körperbewegungen im Sacre mit früheren Nižinskij-Bewegungen bzw. vorherigen Nižinskij-Choreografien zu vergleichen. ${ }^{147}$ Rivière wird so zur historischen Referenz, ein zeitgenössischer Bericht über Nižinskijs Choreografie gewissermaßen zur Choreografie selbst, und Primitivismus und

142 Schwan bezieht sich auf folgende Aussage Nižinskijs: „I am accused of a ,crime against grace', among other things. [...] Really I begin to have horror of the very word; ,grace' and ,charm' make me feel seasick. [...] The fact is, I detest conventional ,nightingale-and-rose poetry; my own inclinations are ,primitive'. I eat my meat without sauce Bérnaise." Vaclav NıžINSKIJ, in: The Daily Mail, 14. Juli 1913.

143 Schwan, „Queer Stupidity“, S. 69. Bezüglich der Rezeption des Sacre im Kontext des zeitgenössischen Primitivismusdiskurses nennt Schwan Buchs 2014 erschienenen Artikel „The Scandal at ,Le Sacre“ als Referenz. Für diesen stellt sich der Primitivismus des Sacre auch vor allem auf der Ebene der Rezeption dar. Vgl. Buch, „The Scandal at ,Le Sacre“, S. $70-78$.

144 Dass Nižinskijs Aussage im Daily Mail-Interview sehr schnell von anderen Medien aufgegriffen wurde und so große Bekanntheit erfuhr, konstatierte 2003 auch Hanna Järvinen und belegte dies mit einigen zeitgenössischen Quellen. Bei ihr findet sich auch, dass Nižinskijs Sauce-Bérnaise-Metapher später von Jacques Rivière in dessen Sacre-Essay aufgegriffen wurde. Vgl. Hanna Järvinen: The Myth of Genius in Movement. Historical Deconstruction of the Nijinsky Legend, PhD Dissertation, University of Turku 2003, S. 344. Rivière beginnt seinen Essay mit folgenden Worten: „La grande nouveauté du ,Sacre du Printemps', c'est le renoncement à la sauce." Rivière, „Le Sacre du Printemps““, 1. November 1913, S. 706.

145 Schwan, „Queer Stupidity“, S. 70.

146 Vgl. ebd., S. 75: „[I]ndem er [gemeint ist Nižinskij] es nach Rivière vermeidet, den Körper unter wehenden Stoffen zu verbergen und in keiner verselbständigten Dynamik versteckt, eröffnet sich gleichzeitig die Möglichkeit, diese Choreografie als bewegtes geometrisches Ornament wahrzunehmen."

147 Vgl. ebd., S. 82: „Laut Rivière zeigt sich ein eckiger Bewegungsstil in Nijnskys eigenen Bewegungen in ,Pétrouchka (1911) ebenso wie in seiner Choreographie für ,l'Après-midi d'un faune' (1912).“ 
Ornament, wie sie Rivière 1913 in Bezug auf den Sacre beschreibt, zur Grundlage einer tanzwissenschaftlichen Reflexion. ${ }^{148}$

Trotz vergleichbarer Ausgangslage zeigen die exemplarisch gewählten Texte in ihren Thesen und Fragestellungen wesentliche Unterschiede auf und verweisen so auf ein jeweils anderes Erkenntnisinteresse: Weiss benutzt die Tatsache, dass der Sacre von der zeitgenössischen Rezeption und später auch von Stravinskij selbst in den Kontext des Primitiven eingeordnet wurde, als Ausgangspunkt für seine allgemeine Reflexion über die Denkfigur des Primitiven, die den westeuropäischen Blick auf das Russische schon vor (aber auch nach) dem Sacre gepägt habe. Schwan hingegen arbeitet nicht klassisch historisch und nutzt Rivière als Referenz für seine Überlegungen zum Sacre selbst.

Es wird deutlich: Sowohl Tanz- als auch Musikwissenschaft nähern sich dem Primitivismusphänomen zu Beginn des 20. Jahrhunderts an, um es in seiner Anwendbarkeit auf die wissenschaftliche Untersuchung musikalischer wie tänzerischer Werke zu prüfen. Und beide Disziplinen weisen hierbei dem Sacre eine besondere Rolle zu. Die beiden beispielhaft vorgestellten Texte deuten allerdings darauf hin, dass von einem geeinten Sacre-Diskurs zwischen Musik- und Tanzwissenschaft schon allein aufgrund des sich unterscheidenden

148 Schwan verweist - wenn er den Begriff Ornament in seinem Aufsatz erstmals gebraucht auf Gabriele Brandstetter: „Die Inszenierung der Fläche. Ornament und Relief im Theaterkonzept der Ballets Russes“, in: Spiegelungen, hg. von Jeschke, Berger und Zeidler, S. 147-163. Brandstetters darin enthaltene allgemeine Ausführungen zur Rolle des Ornaments und dazu, wie es sich in den Produktionen der Ballets Russes äußert, gründen ebenfalls auf einer Beschäftigung mit der Rezeption jener Ballets-Russes-Werke: „Carl Einstein sieht in den textilen Raumgestaltungen von Léon Bakst ein bewegtes Ornament der Bühne: die Draperie eines szenographischen ,Stoffes', einer Textur. Und diese Stoff-Fläche tanzt, [...] der ganze Raum wirbelt, ein rhythmisch bewegtes Farbornament. [...] Im kulturellen Kontext der Jahrhundertwende erscheint diese auf dem Bühnen-Prospekt übertragene Feier der Zweidimensionalität als spezifisch theatrale Variante der Vorliebe für Linie, Dekor und Fläche, wie sie in der Kunst des Jugenstils und Art Deco repräsentiert ist [...]. Im Rahmen dieses kunstgeschichtlichen Szenarios [...] lassen sich drei Konzepte einer Inszenierung der Fläche abgrenzen, die als neue Paradigmen die Wahrnehmung von Raum, Körper und Bewegung steuern [...]. Alle drei dieser Paradigmen einer Ästhetik der Fläche werden gleichsam zu Transformatoren zwischen unterschiedlichen Medien, Darstellungskonventionen und Kulturmustern [...] und zeichnen sich in eigentümlicher Weise in den Produktionen der ,Ballets Russes" ein." Ebd., S. 149f. Ästhetische Paradigmen, die durch Rezeptionsbeobachtungen aufgestellt werden können, werden sowohl bei Schwan als auch bei Brandstetter aufgegriffen und an einem spezifischen Werk, das diesem ästhetischen Paradigma unterstellt werden kann, reflektiert. Brandstetters Referenz ist hier Carl Einste in: „Léon Bakst“, in: Carl Einstein. Werke, Bd. 2: 1919-1928, hg. von Marion SCHMID unter Mitarbeit von Henriette BEESE und Jens KwASNY, Berlin 1981, S. $341-368$, hier S. 358 . 
Erkenntnisinteresses (noch) nicht gesprochen werden kann. Dennoch können das Nebeneinander eines musik- und tanzwissenschaftlichen Textbeitrags zum Sacre in einem interdisziplinären Sammelband zum Phänomen des Primitivimus sowie das Integrieren eines musikwissenschaftlichen Beitrags in einer kulturwissenschaftlichen Studie als ein erster Schritt der Annäherung begriffen werden. 